\title{
Activation of Schwann cells in vitro by magnetic nanocomposites via applied magnetic field
}

\author{
This article was published in the following Dove Press journal: \\ International Journal of Nanomedicine \\ 17 December 2014 \\ Number of times this article has been viewed
}

\author{
Zhongyang Liu',* \\ Liangliang Huang ${ }^{1, *}$ \\ Liang Liu',* \\ Beier Luo ${ }^{2, *}$ \\ Miaomiao Liang $^{3}$ \\ Zhen Sun' \\ Shu Zhu' \\ Xin Quan' \\ Yafeng Yang' \\ Teng $\mathrm{Ma}^{\prime}$ \\ Jinghui Huang' \\ Zhuojing Luo' \\ 'Department of Orthopaedics, Xijing \\ Hospital, Fourth Military Medical \\ University, Xi'an, ${ }^{2}$ Department \\ of Orthopaedics, Changhai \\ Hospital, Second Military Medical \\ University, Shanghai, ${ }^{3}$ Department \\ of Neurosurgery, Xijing Hospital, \\ Fourth Military Medical University, \\ Xi'an, People's Republic of China \\ *These authors contributed equally \\ to this work
}

\begin{abstract}
Schwann cells (SCs) are attractive seed cells in neural tissue engineering, but their application is limited by attenuated biological activities and impaired functions with aging. Therefore, it is important to explore an approach to enhance the viability and biological properties of SCs. In the present study, a magnetic composite made of magnetically responsive magnetic nanoparticles (MNPs) and a biodegradable chitosan-glycerophosphate polymer were prepared and characterized. It was further explored whether such magnetic nanocomposites via applied magnetic fields would regulate SC biological activities. The magnetization of the magnetic nanocomposite was measured by a vibrating sample magnetometer. The compositional characterization of the magnetic nanocomposite was examined by Fourier-transform infrared and $\mathrm{X}$-ray diffraction. The tolerance of SCs to the magnetic fields was tested by flow-cytometry assay. The proliferation of cells was examined by a 5-ethynyl-2-deoxyuridine-labeling assay, a PrestoBlue assay, and a Live/Dead assay. Messenger ribonucleic acid of BDNF, GDNF, NT-3, and VEGF in SCs was assayed by quantitative real-time polymerase chain reaction. The amount of BDNF, GDNF, NT-3, and VEGF secreted from SCs was determined by enzymelinked immunosorbent assay. It was found that magnetic nanocomposites containing $10 \%$ MNPs showed a cross-section diameter of $32.33 \pm 1.81 \mu \mathrm{m}$, porosity of $80.41 \% \pm 0.72 \%$, and magnetization of $5.691 \mathrm{emu} / \mathrm{g}$ at $8 \mathrm{kOe}$. The $10 \% \mathrm{MNP}$ magnetic nanocomposites were able to support cell adhesion and spreading and further promote proliferation of SCs under magnetic field exposure. Interestingly, a magnetic field applied through the 10\% MNP magnetic scaffold significantly increased the gene expression and protein secretion of BDNF, GDNF, NT-3, and VEGF. This work is the first stage in our understanding of how to precisely regulate the viability and biological properties of SCs in tissue-engineering grafts, which combined with additional molecular factors may lead to the development of new nerve grafts.
\end{abstract}

Keywords: Schwann cell, magnetic field, nanocomposite, cell proliferation

\section{Introduction}

In the peripheral nervous system, Schwann cells (SCs) are the main gliocyte type, and undergo dedifferentiation and proliferation in the distal injured segment after injury. They form Büngner bands at injury sites, which create comfortable milieus for axon regeneration and ensuing remyelination. ${ }^{1}$ Multiple factors, including neurotrophic factors, hormones, and extracellular matrix protein, participate in this process. ${ }^{2}$ Driven by these considerations, the combination of SCs with nerve scaffolds has been becoming a more and more attractive therapy to enhance nerve regeneration after nerve injuries. ${ }^{3-6}$ In these cases, a considerable number of SCs with enhanced cell viability and biological properties would be required. However, attenuated biological activities have been found in SC cultures, which significantly limit the efficiency of SCs in nerve-injury repair. ${ }^{7}$ Therefore, it is important to explore an approach to enhance the viability and biological properties of SCs.
Correspondence: Jinghui Huang/ Zhuojing Luo

Department of Orthopaedics, Xijing Hospital, Fourth Military Medical University, 169 Changle West Road, Xi'an, Shaanxi 7I0032, People's Republic of China

Tel +862984775285

Fax +862984775285

Email huangjh@fmmu.edu.cn/zjluo@

fmmu.edu.cn
International Journal of Nanomedicine 20|5:10 43-6I

Dovepress

http://dx.doi.org// 0.2147/IJN.S74332 (c) (i) (5) 2015 Liu et al. This work is published by Dove Medical Press Limited, and licensed under Creative Commons Attribution - Non Commercial (unported, v3.0) License. The full terms of the License are available at http://creativecommons.org/licenses/by-nc/3.0/. Non-commercial uses of the work are permitted without any further permission Love Medical Press Limited, provided the work is properly attributed. Permissions beyond the scope of the License are administered by Dove Medical Press Limited. Information on how to request permission may be found at: http://www.dovepress.com/permissions.php 
Magnetic fields (MFs) have been shown to be safe and effective in regulating cell biological properties. ${ }^{8-10}$ Recently, magnetic composites via applied MFs have been shown to further improve the biological properties of cells. It has been shown that a magnetic composite via MF is capable of stimulating endothelial cell proliferation, promoting organization of endothelial cells into capillary-like structures, ${ }^{11}$ facilitating organization of cardiac cells into myocardial tissue in vitro, ${ }^{12}$ and enhancing osteogenesis for bone repair in vivo. ${ }^{13}$ In a previous study, using MF, regulation of alignment of SCs has been reported. ${ }^{14}$ However, it is unclear whether magnetic nanocomposites via MF can regulate SC biological properties, which is important for the in vitro and in vivo application of SCs.

In the present study, a magnetic nanocomposite with magnetically responsive magnetic nanoparticles (MNPs) was fabricated. We investigated the magnetization of the magnetic nanocomposites. We next examined their compositional and morphological characteristics. In addition, we explored whether the magnetic nanocomposites via MF would promote SC biological activities.

\section{Materials and methods}

\section{Preparation of magnetic nanocomposites}

MNPs were synthesized using an oxidative hydrolysis approach as described previously. ${ }^{15}$ In brief, $1.364 \mathrm{~g} \mathrm{KNO}_{3}$ (99\%; Acros Organics, Belgium) and $0.486 \mathrm{~g} \mathrm{NaOH} \mathrm{(} \geq 99 \%$; Sigma-Aldrich, St Louis, MO, USA) were mixed together, and then the mixture was dissolved in $135 \mathrm{~mL}$ of deionized water bubbled with $\mathrm{N}_{2}$ flow. Then, a solution that had been previously prepared under 3 hours of $\mathrm{N}_{2}$ flow with $15 \mathrm{~mL}$ of $0.01 \mathrm{M} \mathrm{H}_{2} \mathrm{SO}_{4}$ (96\%; Panreac, Spain) solution containing $0.308 \mathrm{~g} \mathrm{FeSO}_{4} \cdot 7 \mathrm{H}_{2} \mathrm{O}$ ( $\geq 99 \%$; Sigma-Aldrich) was added drop by drop under constant stirring. When the precipitation was finished, $\mathrm{N}_{2}$ flow for an additional 10 minutes was allowed to pass, and the temperature was heated to $90^{\circ} \mathrm{C}$ for 24 hours. Finally, the product was cooled to room temperature by means of an ice bath. The synthetic black product was isolated by magnetic decantation, and then rinsed several times using deionized water to remove all residual impurities. The crystallization of MNPs was determined using X-ray diffraction (XRD) by $\mathrm{Cu} \mathrm{K} \alpha \mathrm{X}$-radiation (Rigaku Miniflex, Japan), and the morphology, distribution, and average size of MNPs were examined by transmission electron microscopy (TEM; H-600; Hitachi, Japan). The chemical composition of MNPs was recorded using Fourier-transform infrared (FTIR) spectrophotometry (8400s; Shimadzu, Japan), and spectra were measured in the mid-IR range (128 scans) from 4,000 to $400 \mathrm{~cm}^{-1}$ (at a resolution of $4.0 \mathrm{~cm}^{-1}$ ). Magnetization was determined with a vibrating sample magnetometer (665; Lake Shore Cryotronics, USA).

To prepare magnetic nanocomposites (5\%, 10\%, 20\% w/w MNPs), $5 \mathrm{~mL}$ of $0.1 \mathrm{M}$ acetic acid aqueous solution and $100 \mathrm{mg}$ chitosan powder (molecular weight $>310 \mathrm{kD}$, deacetylation $\approx 90 \%$, Sigma-Aldrich) were mixed well to prepare a chitosan solution. The glycerophosphate salt (Fluka, USA) solution, prepared by adding $1 \mathrm{~mL}$ phosphate-buffered saline (PBS) to $500 \mathrm{mg}$ glycerophosphate salt, was added to the chitosan solution drop-wise with an ice bath. The final solution was homogeneous and clear, with a $\mathrm{pH}$ of 7.04. Then, a chitosan-glycerophosphate mixture was stored at $-80^{\circ} \mathrm{C}$ for 24 hours and lyophilized for another 24 hours. Then, $100 \mathrm{mg}$ chitosan-glycerophosphate lyophilized powder was redissolved in $5 \mathrm{~mL}$ of deionized water, and MNPs were added to the chitosan-glycerophosphate solution with vigorous stirring in an ice bath for 2 hours. Later, the MNP-chitosan mixture was vacuumized for 24 hours at $4^{\circ} \mathrm{C}$ and stored at $4^{\circ} \mathrm{C}$. For different experimental purposes, the MNP-chitosan mixture was fabricated into membranes or scaffolds. For magnetic membranes, the MNP-chitosan mixture was later modeled onto a plastic dish and air-dried at room temperature for 24 hours. The dried magnetic membranes were washed using deionized water to remove spare MNPs, and air-dried again at room temperature. For magnetic scaffolds, the MNP_chitosan mixture was injected into a tailor-made mold, which was immediately stored at $-80^{\circ} \mathrm{C}$ for 24 hours and then lyophilized for 24 hours. Then, the magnetic scaffolds were separated from the mold and cut into cylinders ( $2.0 \mathrm{~cm}$ in length, $2.0 \mathrm{~mm}$ in diameter). The dried magnetic scaffolds were rinsed with deionized water to remove spare MNPs, and lyophilized again for 24 hours. Ethylene dioxide gas was used to sterilize the magnetic membranes and magnetic scaffolds at $37^{\circ} \mathrm{C}$ according to standard industrial procedures.

\section{Magnetic measurements}

Magnetization of magnetic nanocomposites at a low field was detected at 34 Oe via a YSZ 01C/02C susceptometer (Sartorius Mechatronics, Italy). Calibration for high-field magnetization was measured using the sample magnetometer at room temperature. The magnetic potentiality of the magnetic scaffolds was investigated by using an external magnet.

\section{Compositional characterization}

FTIR spectral analysis is necessary for the identification of functional groups present in a chemical product based on their vibration energy. The FTIR spectrum of the magnetic 
mixtures was recorded using FTIR. In parallel, XRD analysis was also performed.

\section{Morphological characterization}

Morphological observation of the magnetic membranes and magnetic scaffolds was performed on each type using scanning electron microscopy (SEM; JSM-4800; Hitachi) at an accelerating voltage of $5 \mathrm{kV}$. The samples had been previously sputter-coated with gold and analyzed under partial vacuum.

\section{Porosity}

The porosity of the magnetic scaffolds was tested by a specific customized bottle (Hubbard; Hanil, South Korea) on the basis of Archimedes's principle. ${ }^{16}$ Briefly, the porosity of scaffolds was calculated as follows:

$$
\begin{gathered}
\text { Porosity }(\%)=100 \% \times \mathrm{V}_{\mathrm{P}} / \mathrm{V}=100 \% \times\left(\left(\mathrm{W}_{1}-\mathrm{W}_{2}-\mathrm{W}\right) / \mathrm{\rho}\right) / \\
\left(\left(\mathrm{W}_{0}-\mathrm{W}_{2}\right) / \mathrm{\rho}\right)=100 \% \times\left(\mathrm{W}_{1}-\mathrm{W}_{2}-\mathrm{W}\right) /\left(\mathrm{W}_{0}-\mathrm{W}_{2}\right)
\end{gathered}
$$

where $\mathrm{V}_{\mathrm{P}}$ is the pore volume in the scaffold, $\mathrm{V}$ is the total volume of the scaffold including pores, $\mathrm{W}_{0}$ is the customized bottle filled with ethanol, $\mathrm{W}_{1}$ is the customized bottle weight including scaffold and ethanol, $\mathrm{W}_{2}$ is the customized bottle weight taken out of the ethanol-loaded scaffold from $\mathrm{W}_{1}$, W is the scaffold weight, and $\rho$ is the density of ethanol; therefore,
$\left(\mathrm{W}_{0}-\mathrm{W}_{2}\right) / \rho$ is the total volume of the scaffold including pores, and $\left(\mathrm{W}_{1}-\mathrm{W}_{2}-\mathrm{W}\right) / \rho$ is the pore volume in the scaffold.

\section{SC culture and identification}

SCs were prepared and purified following a protocol described previously. ${ }^{17}$ All the experimental procedures were performed in accordance with the Guide for the Care and Use of Laboratory Animals. ${ }^{18}$ In brief, SCs were isolated from the sciatic nerves of 1- to 2-day-old Sprague Dawley rats (provided by the Experimental Animal Center of the Fourth Military Medical University) and further selected from fibroblasts using fibronectin-specific antibody and rabbit complement. The purity of the SCs was determined by double-immunofluorescent staining with S-100 (red) and Sox-10 (green). The numbers of S-100- and Sox-10-positive cells and 4',6-diamidino-2-phenylindole (DAPI)-labeled cells were compared. The final preparations consisted of a high purity ( $>96 \%$ ) of SCs (Figure 1). SCs were maintained in Dulbecco's Modified Eagle's Medium nutrient mixture F-12 (DMEM/F12; Gibco, USA) containing 10\% fetal calf serum (FCS; Gibco), antibiotics $(100 \mathrm{UI} / \mathrm{mL}$ penicillin and 100 $\mu \mathrm{g} / \mathrm{mL}$ streptomycin solution), $20 \mu \mathrm{g} / \mathrm{mL}$ bovine pituitary extract (Biomedical Technologies, USA) and $2 \mu \mathrm{M} / \mathrm{mL}$ forskolin (Sigma-Aldrich) at $37^{\circ} \mathrm{C}$ under humidified $5 \% \mathrm{CO}_{2}$. $\mathrm{SC}$ cultures were passaged no more than five times before conducting experiments.
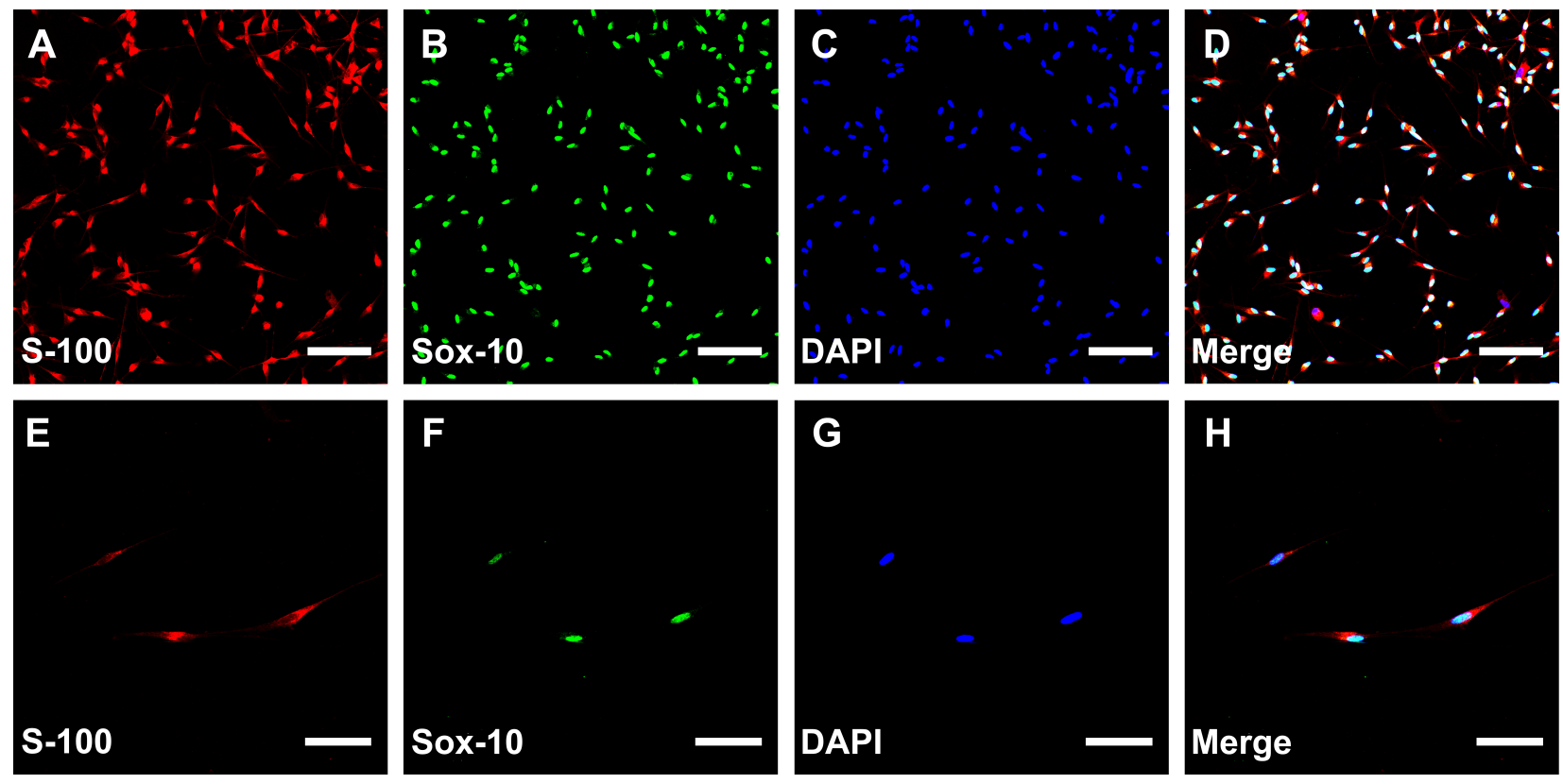

Figure I Characterization of SCs.

Notes: Double-immunofluorescent staining showed the expression of S-100 (A and E) and Sox-10 (B and F) with DAPI nuclear counterstaining (C and G). Merge image showed a purity of more than $96 \%$ SCs (D and H). Scale bars: (A-D) $100 \mu \mathrm{m}$ (magnification 20x), (E-H) $50 \mu \mathrm{m}$ (magnification 40X).

Abbreviations: SCs, Schwann cells; DAPI, 4',6-diamidino-2-phenylindole. 


\section{Magnetic exposure system}

The magnetic exposure system comprised a solenoid and a MF generator to produce MFs with adjustable magnetic induction of $0-20 \mathrm{mT}$ and frequency of $0-100 \mathrm{~Hz}$. The dimensions of the solenoid were $40 \mathrm{~mm}$ high, $400 \mathrm{~mm}$ long, $100 \mathrm{~mm}$ inner diameter, and $200 \mathrm{~mm}$ outer diameter (Figure 2B). This customized solenoid was wrapped with $1.00 \mathrm{~mm}$-diameter enamel-coated copper wire (500 turns). A MF generator (GHY-III, patent ZL02224739.4; FMMU, People's Republic of China [PRC]) connected with the solenoid to produce an open-circuit output waveform MF. The measurement accuracy of the electromagnetic field output was confirmed with a Gaussmeter (Model 455 DSP; Lake Shore Cryotronics). A small $2 \Omega$ resistor was laid correctly in series with the solenoid, and the wave shape and frequency were visualized by an oscilloscope (6000 series; Agilent Technologies, USA). The MF parameter was set to a frequency of $50 \mathrm{~Hz}$, as described in previous experiments. ${ }^{9,19,20}$ In the nonstimulated group, the cell-culture plate was put correctly in a solenoid that was connected to the MF generator, but had no output waveform.

\section{Nanocomposites prepared for biological testing}

Only 10\% MNP magnetic membranes (thickness, $0.4 \mathrm{~mm}$ ) and 10\% MNP magnetic scaffolds were used for biological testing. Nonmagnetic membranes and nonmagnetic scaffolds were also used as controls. All membranes were cut into rectangular sections $(76 \times 25 \mathrm{~mm})$. The membrane sections were fastened to the bottom of the customized magnetic cellculture plate. All scaffolds were cut into cylinders $(5.0 \mathrm{~mm}$ in height, $2.0 \mathrm{~mm}$ in diameter).

\section{Magnetic exposure of SCs}

When SCs reached $95 \%$ confluence, a $0.05 \%$ trypsin solution was used to dissociate SCs, and then they were rinsed gently

A

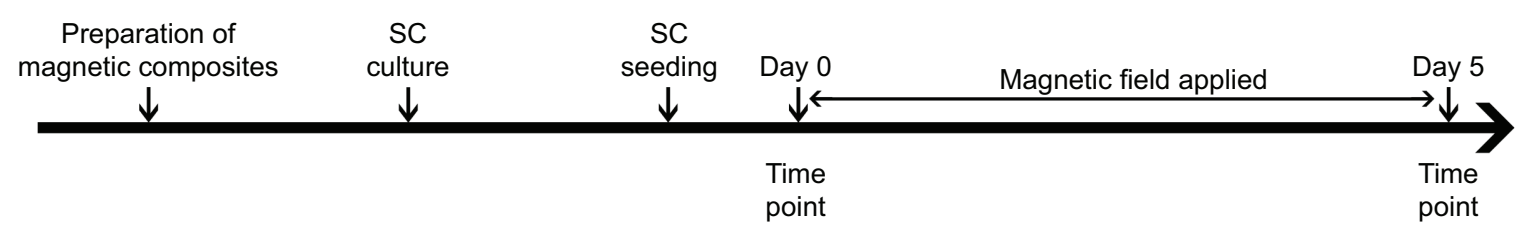

B

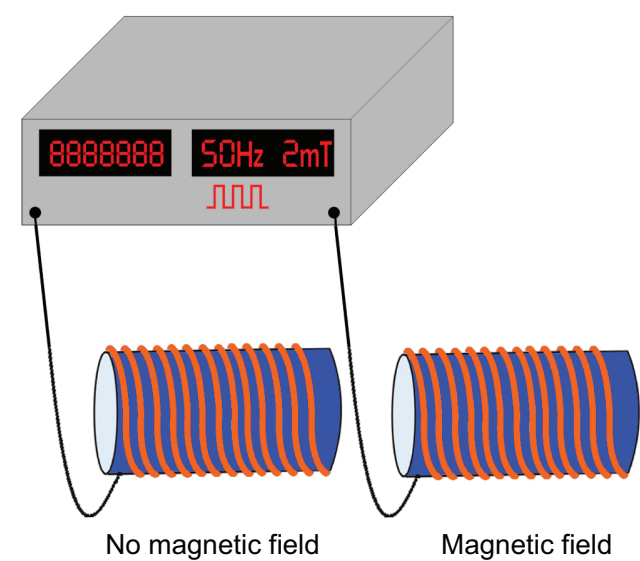

D

(1)

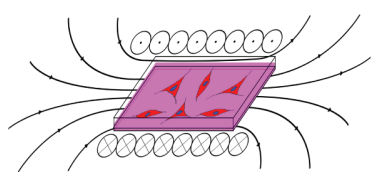

C
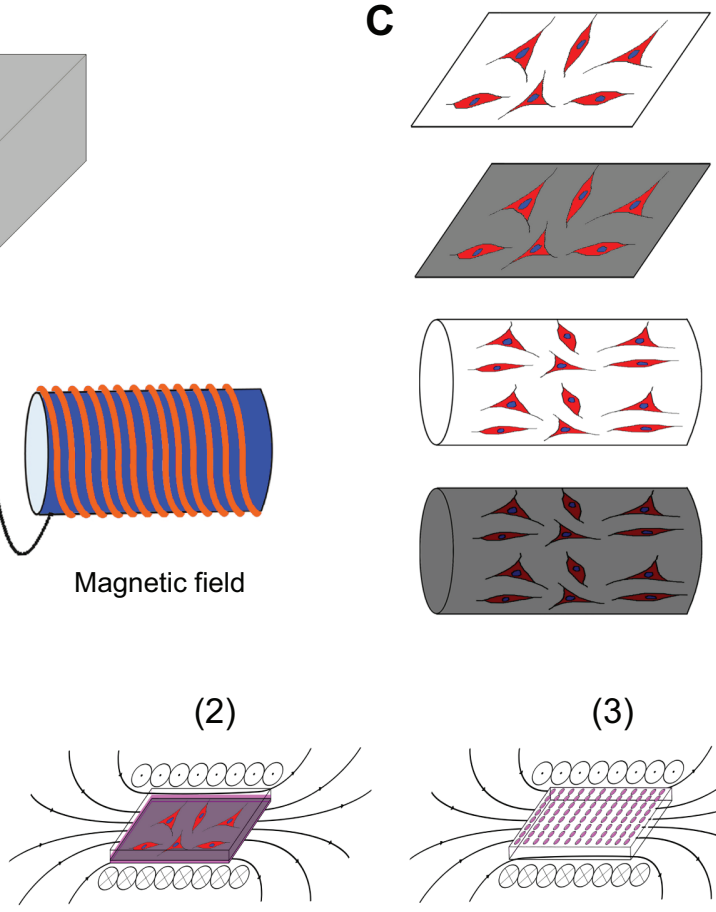

(3)

(2)

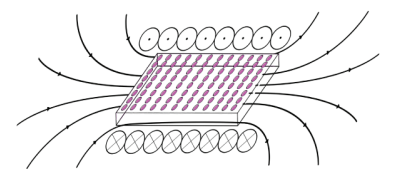

SCs seeded on nonmagnetic membrane

SCs seeded on magnetic membrane

SCs cocultivated with nonmagnetic scaffold

SCs cocultivated with magnetic scaffold

Figure 2 Schematic view of this experiment.

Notes: (A) Experimental time scale. (B) The device used to expose SCs to the MF and the no-MF stimulations. The solenoid was wired to a pulse generator. (C) The membrane and scaffold seeded with SC model. (D) The distribution of the MF in the solenoid. Models were placed in the axial plane of the solenoid.

Abbreviations: SCs, Schwann cells; MF, magnetic field. 
and resuspended in DMEM/F12 containing 10\% FCS. The cells were later seeded on 10\% MNP magnetic membranes, nonmagnetic membranes, and culture plates, respectively, and then incubated for cell settling and adhesion within 24 hours. Then, different MF gradients $(0.5 \mathrm{mT}, 1 \mathrm{mT}, 2 \mathrm{mT}, 5 \mathrm{mT}$, $10 \mathrm{mT} ; 50 \mathrm{~Hz}$ ) were applied to the cells seeded on the membrane for 2 hours using the MF-exposure system described in the Magnetic exposure system section. After MF exposure, fresh DMEM/F12 with $10 \%$ FCS was used to replace the culture medium. The cells were then cultured for an additional 24 hours. Cells cultured on magnetic membranes without MF exposure, nonmagnetic membranes without MF exposure, and culture plates without MF exposure served as controls. For stereoscopic cultivation, the cells $\left(1 \times 10^{4}\right.$ cells/well $)$ were cocultivated with pieces of the $10 \%$ MNP magnetic scaffolds and nonmagnetic scaffolds in 96-well plates for 24 hours to allow settling and adhesion. MF exposure was then applied to the cells for 2 hours. After MF exposure, fresh DMEM/F12 containing $10 \%$ FCS was used to replace the culture medium. The cells were cultured for an additional 24 hours. Cells cocultivated with magnetic scaffolds without MF exposure, nonmagnetic scaffolds without MF exposure, and culture plates without MF exposure served as controls.

\section{Flow-cytometry assay}

The SCs $\left(1 \times 10^{5}\right.$ cells $\left./ \mathrm{cm}^{2}\right)$ were cultured on the $10 \% \mathrm{MNP}$ magnetic membranes, nonmagnetic membranes, and culture plates, respectively, for 24 hours, and then different MF gradients $(0.5 \mathrm{mT}, 1 \mathrm{mT}, 2 \mathrm{mT}, 5 \mathrm{mT}, 10 \mathrm{mT}$; $50 \mathrm{~Hz})$ were applied to the cells seeded on the membranes for 2 hours. After MF exposure, SCs were cultured for another 24 hours, and then the percentage of viable cells was analyzed by flow cytometry. All samples were analyzed within 30 minutes.

\section{CCK-8 assay}

The viability of SCs seeded on magnetic membranes was detected and quantified using the Cell Counting Kit 8 (CCK-8; Dojindo, Japan). Briefly, the SCs seeded on magnetic membranes were under different exposure times $(0.5$, $1,2,3$, and 4 hours) of the MF (2.0 mT). At 24 and 48 hours after MF exposure, SCs were washed three times with PBS. Then, SCs were detached from the magnetic membrane using a $0.05 \%$ trypsin solution, washed, and resuspended in DMEM/F12. Thereafter, the cell suspension was inoculated $(100 \mu \mathrm{L} /$ well) in a $96-w e l l$ plate. The CCK-8 solution $(10 \mu \mathrm{L})$ was added to each well of the plate and incubated for 4 hours. The absorbance was measured at $492 \mathrm{~nm}$ using a microplate reader.

\section{Evaluation of SC morphology on membranes and scaffolds}

Twenty-four hours after MF stimulation, the adhesion of SCs in each group was detected with SEM. In brief, SCs were rinsed with PBS twice and then fixed with 4\% paraformaldehyde for 25 minutes at room temperature, washed three times using deionized water, and then dehydrated with serial ethanol solutions, followed by brief vacuum-drying. Thereafter, the specimens were sputter-coated with gold, and then observed by SEM. The dendritic segments of SCs on membranes were chosen randomly from apical and basal regions, and at least one soma's length away from the cell soma, the length of randomly selected dendritic segments was measured.

\section{PrestoBlue assay}

Cell viability was analyzed using a PrestoBlue ${ }^{\mathbb{R}}$ cell-viability reagent (Life Technologies, USA) according to the procedures given by the manufacturer. PrestoBlue is a nontoxic aqueous fluorescent dye that does not affect the viability, phenotype, or proliferation of cells. In brief, 24 hours after MF stimulation, the membranes with cells seeded were rinsed gently with sterilized PBS. Then, SCs were trypsinized, washed, and resuspended in DMEM/F12. Thereafter, $90 \mu \mathrm{L}$ of the cell suspension was inoculated in each well of a 96-well plate. Then, $10 \mu \mathrm{L}$ of the PrestoBlue reagent was added to each well. The plate was then cultured for another 120 minutes. The cells cocultivated with the scaffolds were also tested using the PrestoBlue reagent according to the same method. Twenty-four hours after MF exposure, the medium was replaced with $90 \mu \mathrm{L}$ of fresh serum-free DMEM/F12 and $10 \mu \mathrm{L}$ of PrestoBlue reagent. The plate was then cultured for another 120 minutes, and then the media were transferred to a separate $96-$ well plate for measuring. Optical density readings were collected at 570/630 $\mathrm{nm}$ using a microplate reader and compared to culture plate-group cells. A calibration curve was prepared using the data collected from the wells, which contained known numbers of viable cells.

\section{EdU-labeling assay on membranes}

Cell-proliferation assays were performed using the CellLight $^{\mathrm{TM}}$ 5-ethynyl-2-deoxyuridine (EdU) deoxyribonucleic acid (DNA) cell-proliferation kit (RiboBio, PRC) based on the manufacturer's protocol. In brief, 24 hours after MF exposure, EdU was applied 4 hours prior to fixation of SCs and the EdU immunostaining. To determine the proliferation level of SCs, an EdU-labeling index was calculated as the amount of EdU-stained nuclei divided by the amount 
of DAPI-stained nuclei calculated from five random fields. Assays were done three times using triplicate wells.

\section{Live/Dead assays on scaffolds}

For assessing the survival and proliferation of SCs cocultivated with scaffolds, Live/Dead (BioVision, USA) assays were performed, which employed two color fluorescent dyes (Live/Dead cell-staining kit). Live cells were stained by Live-Dye ${ }^{\mathrm{TM}}$, a green fluorescent dye (excitation/ emission $488 / 518 \mathrm{~nm}$ ), and dead cells were stained by propidium iodide, a red fluorescent dye (excitation/emission 488/615 nm). For the Live/Dead assay, MF exposure was applied to cells for 2 hours every day at the same time point, and then the cells were incubated for an additional 22 hours. Briefly, on day 1 , day 3, and day 5 after MF exposure, the SC scaffolds were incubated with $200 \mu \mathrm{L}$ of fresh serumfree DMEM/F12 with $5 \mathrm{mM}$ of Live-Dye and $5 \mathrm{mM}$ of propidium iodide. After incubation $\left(2\right.$ hours at $37^{\circ} \mathrm{C}$ and $5 \% \mathrm{CO}_{2}$ ), the SC scaffolds were washed three times with sterilized PBS at 5-minute intervals to remove unbound reaction products. The bicolor labeling was visualized using a microscope (FV-1000, Olympus, Japan) equipped for fluorescent detection and fitted with a digital chargecoupled-device camera for image capturing (Optronics, USA). An FV10-ASW 3.1 viewer (Olympus, Japan) was used for image analysis.

\section{Gene-expression analysis}

At 12, 24, and 36 hours after MF exposure, SCs both in membranes and scaffolds were first counted and then homogenized in Trizol reagent (Sigma-Aldrich). Total ribonucleic acid (RNA) was isolated and normalized to cell numbers. Complementary DNA was synthesized using Superscript III reagents according to the manufacturer's instructions (Invitrogen, US). Then, quantitative real-time PCR (QRT-PCR) analysis was performed. The sequencers of primers for $B D N F$, $G D N F, N T-3, V E G F-A$, and $A C T B$ (internal control) are shown in Table 1. QRT-PCR conditions were as follows: denaturation at $95^{\circ} \mathrm{C}, 30$ seconds; primer annealing at $59^{\circ} \mathrm{C}, 30$ seconds; and elongation at $72^{\circ} \mathrm{C}, 40$ seconds. Quantification of PCR products was performed using the $2-\Delta \Delta C$ t method. Quantities of messenger RNA (mRNA) were normalized to the housekeeping gene $A C T B$. Assays were done three times using triplicate wells.

\section{ELISA assay}

For enzyme-linked immunosorbent assay (ELISA) analysis, the SCs were cultured in fresh DMEM/F12 medium without FCS. At 12, 24, and 36 hours after MF exposure, the number of SCs was counted, and the amount of BDNF, GDNF, NT-3, and VEGF secreted by the cultured SCs was determined by the collected culture mediums. Cell-culture supernatants were centrifuged, and the assays were carried out using an ELISA kit (Antibodies Online, Germany). The absorbance of each well was measured using a micro-ELISA reader (Multiscan MK3; Thermo Labsystems, Finland) at $450 \mathrm{~nm}$. Then, the amount of BDNF, GDNF, NT-3, and VEGF secreted by SCs was normalized to cell numbers in each group.

\section{Statistical analysis}

All data presented here are expressed as means \pm standard error of the mean. The data were analyzed using one-way analysis of variance with SPSS 13.0 software (SPSS, USA). Values of $P<0.05$ were considered statistically significant.

\section{Results}

\section{Characteristic determination of MNPs}

The morphology of the MNPs was observed by TEM. MNPs were relatively uniform in size (Figure $3 \mathrm{~A}$ ), and the mean size of MNPs was $28.44 \pm 8.73 \mathrm{~nm}$ (Figure 3B). XRD showed that the crystalline pattern of MNPs was almost in accordance

Table I Primer sequences used for real-time polymerase chain reaction

\begin{tabular}{|c|c|c|c|c|}
\hline Gene & $\begin{array}{l}\text { GenBank accession } \\
\text { number }\end{array}$ & Direction & Sequence & Length (bp) \\
\hline \multirow[t]{2}{*}{ BDNF } & NM_0I25I3.4 & Upper & 5' AGTATTAGCGAGTGGGTC $3^{\prime}$ & 189 \\
\hline & & Lower & 5' GTTCCAGTGCCTTTTGTC 3' & \\
\hline \multirow[t]{2}{*}{ GDNF } & NM_019139.I & Upper & 5' CAGAGGGAAAGGTCGCAGAG 3' & 114 \\
\hline & & Lower & 5' ATCAGTTCCTCCTTGGTTTCGTAG 3' & \\
\hline \multirow[t]{2}{*}{ NTF3 } & NM_00I270870 & Upper & 5' GCAGGGTGAAGGGGAAAAC 3' & 254 \\
\hline & & Lower & 5' GCCACGGAGATAAGCAAGAAA 3' & \\
\hline \multirow[t]{2}{*}{ VEGFA } & NM_031836.2 & Upper & 5' AACTTCTACCCGTGCCTT 3' & 233 \\
\hline & & Lower & 5' ACTTAGGTCAGCGTTTCC $3^{\prime}$ & \\
\hline \multirow[t]{2}{*}{ ACTB } & NM_03II 44.2 & Upper & 5' ATGAAGATCCTGACCGAG 3' & 193 \\
\hline & & Lower & 5' GCTCATTGCCGATAGTGA 3' & \\
\hline
\end{tabular}



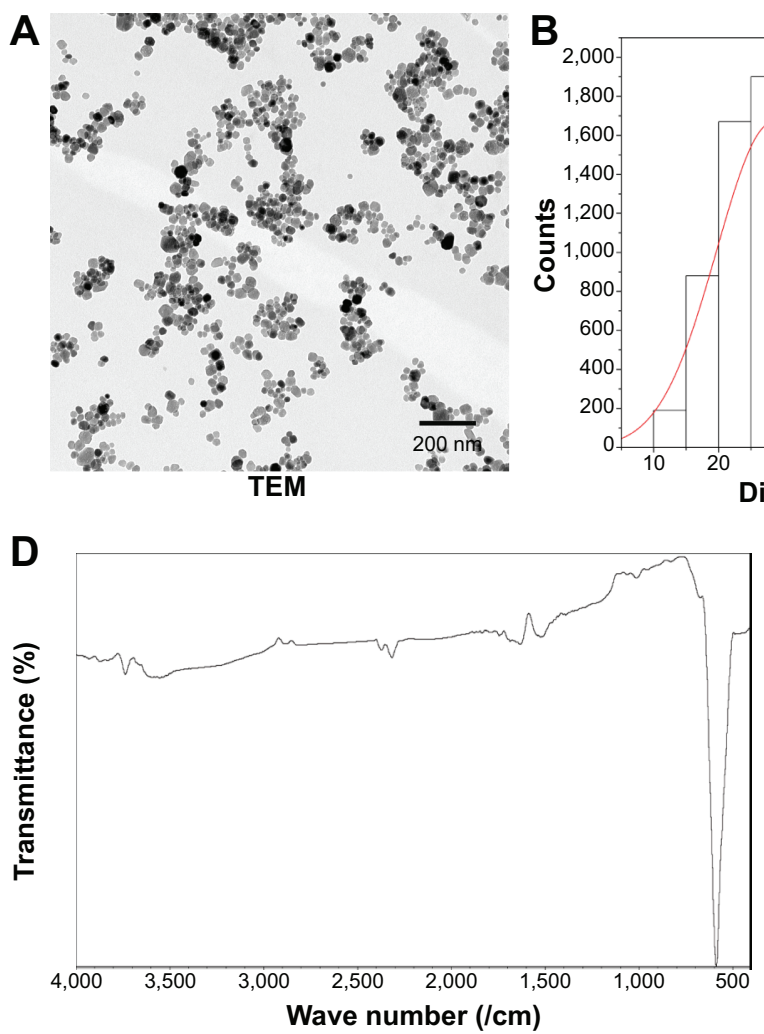
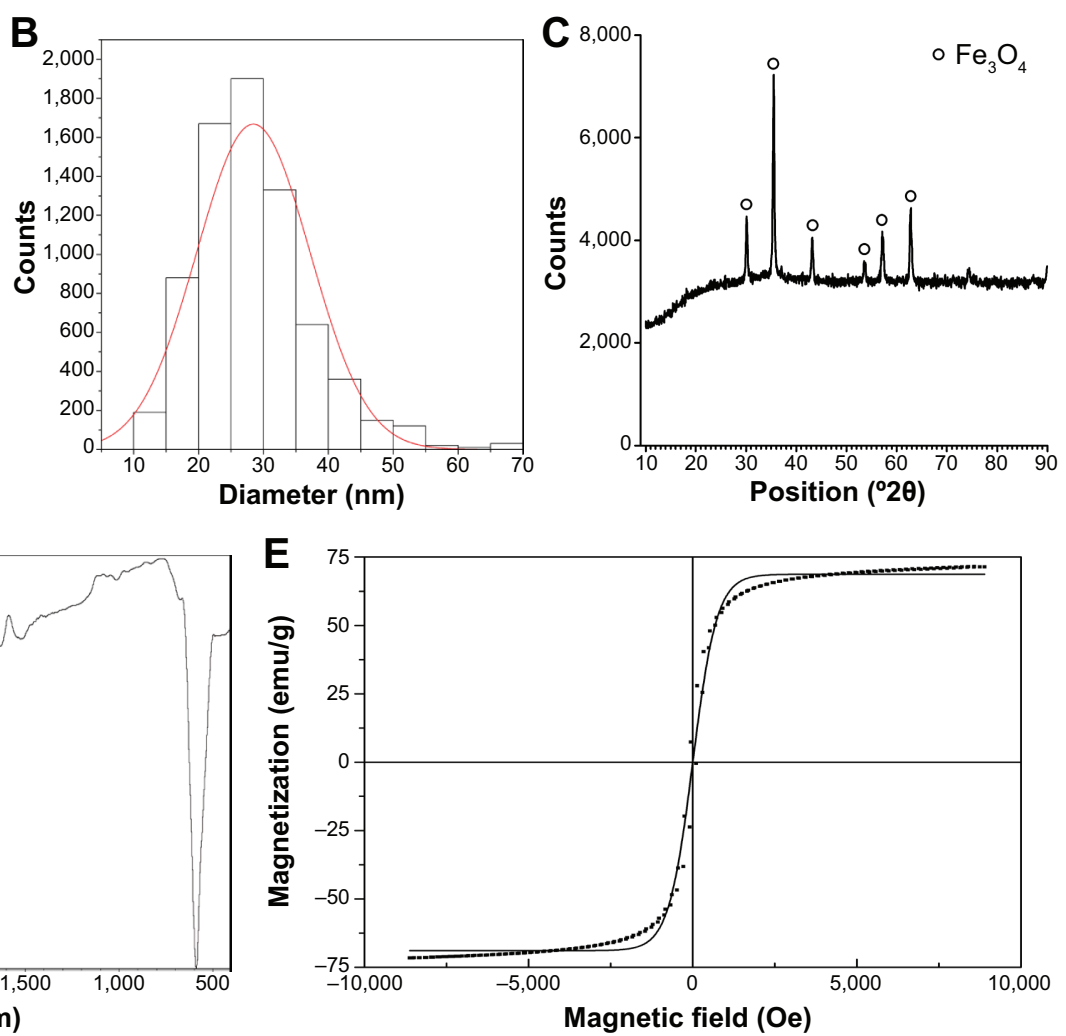

Figure 3 Characterization of MNPs.

Notes: (A) TEM. (B) Size distribution of MNPs. (C) XRD. (D) FTIR spectrum. (E) Magnetization of MNPs.

Abbreviations: MNPs, magnetic nanoparticles; TEM, transmission electron microscopy; XRD, X-ray diffraction; FTIR, Fourier-transform infrared.

with standard iron oxide crystals (Figure 3C). ${ }^{21}$ The FTIR spectrum showed that the characteristic absorption peak for iron oxide was measured at $584 \mathrm{~cm}^{-1}$ (Figure 3D), which was the specific $\mathrm{Fe}-\mathrm{O}$ vibration. In addition, MNPs showed superparamagnetism (Figure 3E).

\section{FTIR and XRD}

The FTIR spectra of the magnetic nanocomposites and chitosan-glycerophosphate mixture are shown in Figure 4A. The FTIR spectrum of the chitosan-glycerophosphate mixture showed a broad absorption band in the range of $3,600-3,000 \mathrm{~cm}^{-1}$, which was attributable to the overlap of $v(\mathrm{OH})$ and $v(\mathrm{NH})$ stretching. A smaller absorption band at $2,880 \mathrm{~cm}^{-1}$ was due to $v(\mathrm{CH})$ stretching. The characteristic bands at 1,650 and $1,560 \mathrm{~cm}^{-1}$ resulted from $v(\mathrm{C}=\mathrm{O})$ stretching and $\delta(\mathrm{N}-\mathrm{H})$ bending, respectively. The FTIR spectrum exhibited double peaks at 1,070 and $1,000 \mathrm{~cm}^{-1}$ for $\mathrm{v}(\mathrm{C}-\mathrm{O}-\mathrm{C})$ stretching. In addition, a broad band in the range of $600-500 \mathrm{~cm}^{-1}$ (bending motion of the $\mathrm{O}=\mathrm{C}-\mathrm{N}$ groups) was also observed. The characteristic of the $\mathrm{Fe}-\mathrm{O}$ vibration was not measured, because of the low content of MNPs in the nanocomposites. In addition, its characteristic absorption peak drowned in the chitosan-glycerophosphate absorption band. The XRD-specific spectrum of MNPs was observed in the magnetic nanocomposites (Figure 4B). The FTIR and XRD spectra of all the magnetic nanocomposites were similar, although they differed slightly from one another due to different weight ratios of MNPs.

\section{Magnetic characterization}

The magnetic properties of magnetic nanocomposites under MF were determined for $M_{L}$, which represents the magnetization measured at $34 \mathrm{Oe}$ (low MF), and for $\mathrm{M}_{\mathrm{H}}$, which represents the magnetization measured at $8 \mathrm{kOe}$ (high MF). The magnetic nanocomposites showed strong $\mathrm{M}_{\mathrm{L}}$, with $0.091 \mathrm{emu} / \mathrm{g}$ in $5 \%$ MNP magnetic nanocomposite, $0.173 \mathrm{emu} / \mathrm{g}$ in $10 \% \mathrm{MNP}$ magnetic nanocomposite, and $0.256 \mathrm{emu} / \mathrm{g}$ in $20 \% \mathrm{MNP}$ magnetic nanocomposite, respectively. The nanocomposites showed superparamagnetism at room temperature (Figure 5B). The $\mathrm{M}_{\mathrm{H}}$ was $1.721 \mathrm{emu} / \mathrm{g}$ in 5\% MNP magnetic nanocomposite, $5.691 \mathrm{emu} / \mathrm{g}$ in 10\% MNP magnetic nanocomposite, and $8.063 \mathrm{emu} / \mathrm{g}$ in $20 \% \mathrm{MNP}$ magnetic nanocomposite, respectively. The magnetic property of magnetic scaffolds was confirmed using a magnet (Figure 5, C and D). The magnetic scaffolds were attracted by the magnet in all directions (updown, in-out), rather than nonmagnetic scaffolds. 

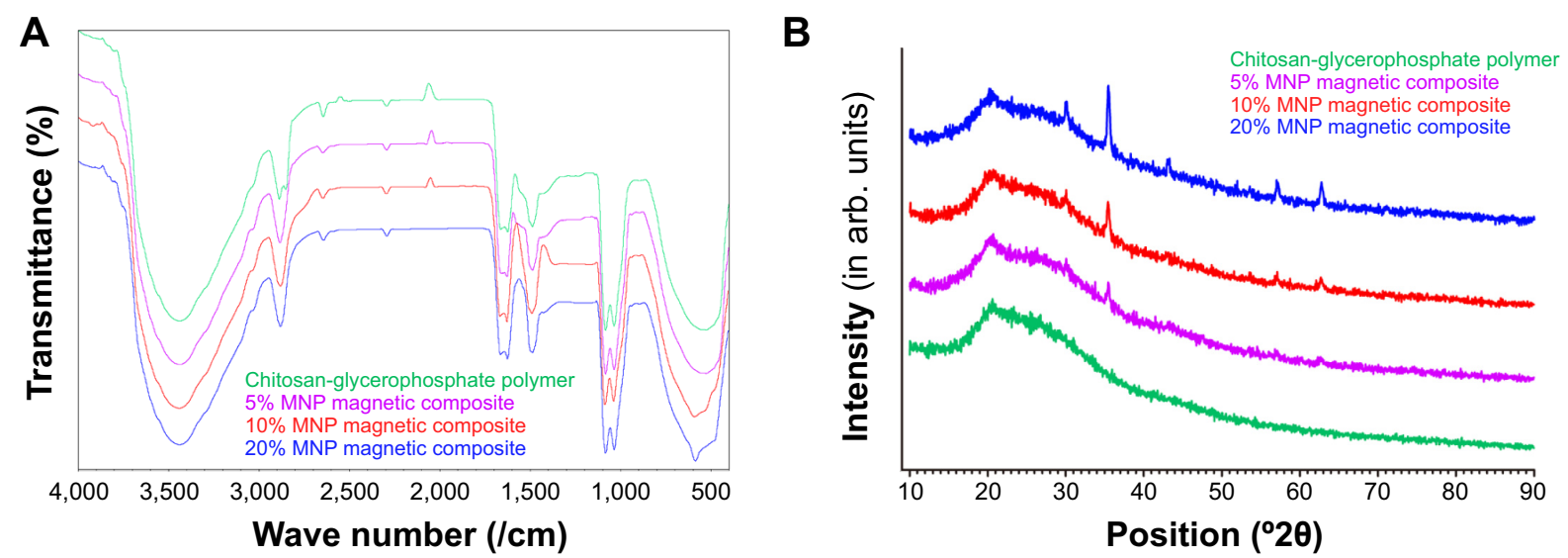

Figure 4 FTIR spectrum and XRD images of the magnetic nanocomposites.

Notes: The FTIR (A) and XRD (B) spectra of all the magnetic nanocomposites were similar, although they differed slightly from each other due to different weight ratios of MNPs.

Abbreviations: FTIR, Fourier-transform infrared; XRD, X-ray diffraction; MNPs, magnetic nanoparticles.

\section{Morphology of magnetic membranes and magnetic scaffolds}

SEM was introduced to study the morphology of magnetic membranes and magnetic scaffolds. The surface of the membranes was neat, and showed almost no cracks (Figure 6, A-D).
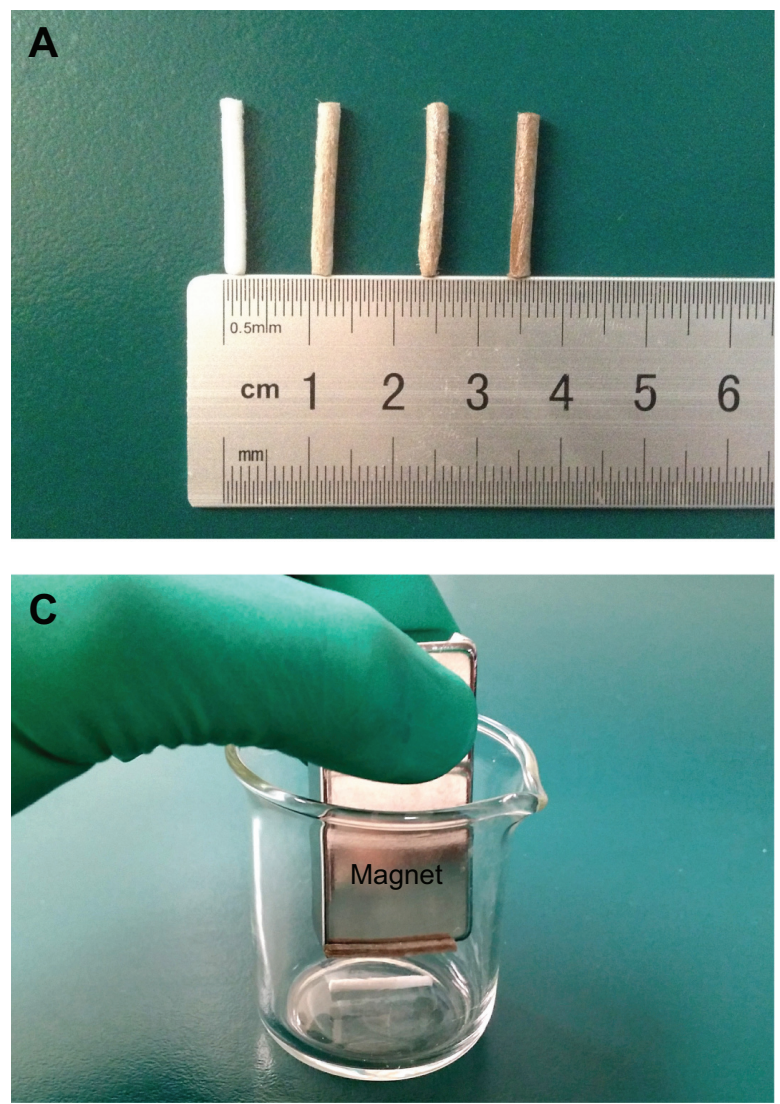

All scaffolds displayed lengthwise-oriented microchannels with various lengths in the longitudinal section (Figure 6, E-L), and a honeycomb-like pattern of the microchannels was arranged in the cross section (Figure 6, M-P). The average cross-sectional diameter of different-content magnetic
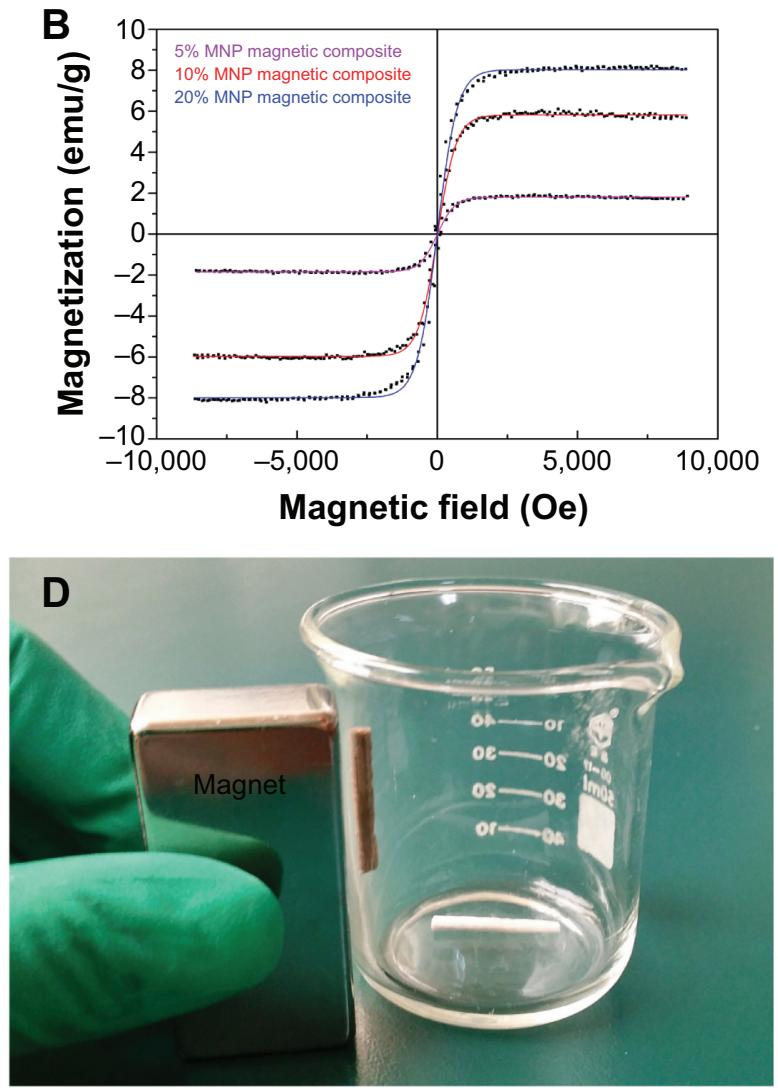

Figure 5 The appearance and magnetization of magnetic scaffolds.

Notes: (A) The appearance of magnetic scaffolds. From left to right: nonmagnetic scaffold, $5 \%$ MNP magnetic scaffold, $10 \%$ MNP magnetic scaffold, and $20 \%$ MNP magnetic scaffold. (B) The magnetization of magnetic nanocomposites was tested with a VSM. Photographs showing the attraction of the $10 \%$ MNP magnetic scaffold to a standard magnet after magnetization: up-down (C), and in-out (D).

Abbreviations: MNP, magnetic nanoparticle; VSM, vibrating sample magnetometer. 

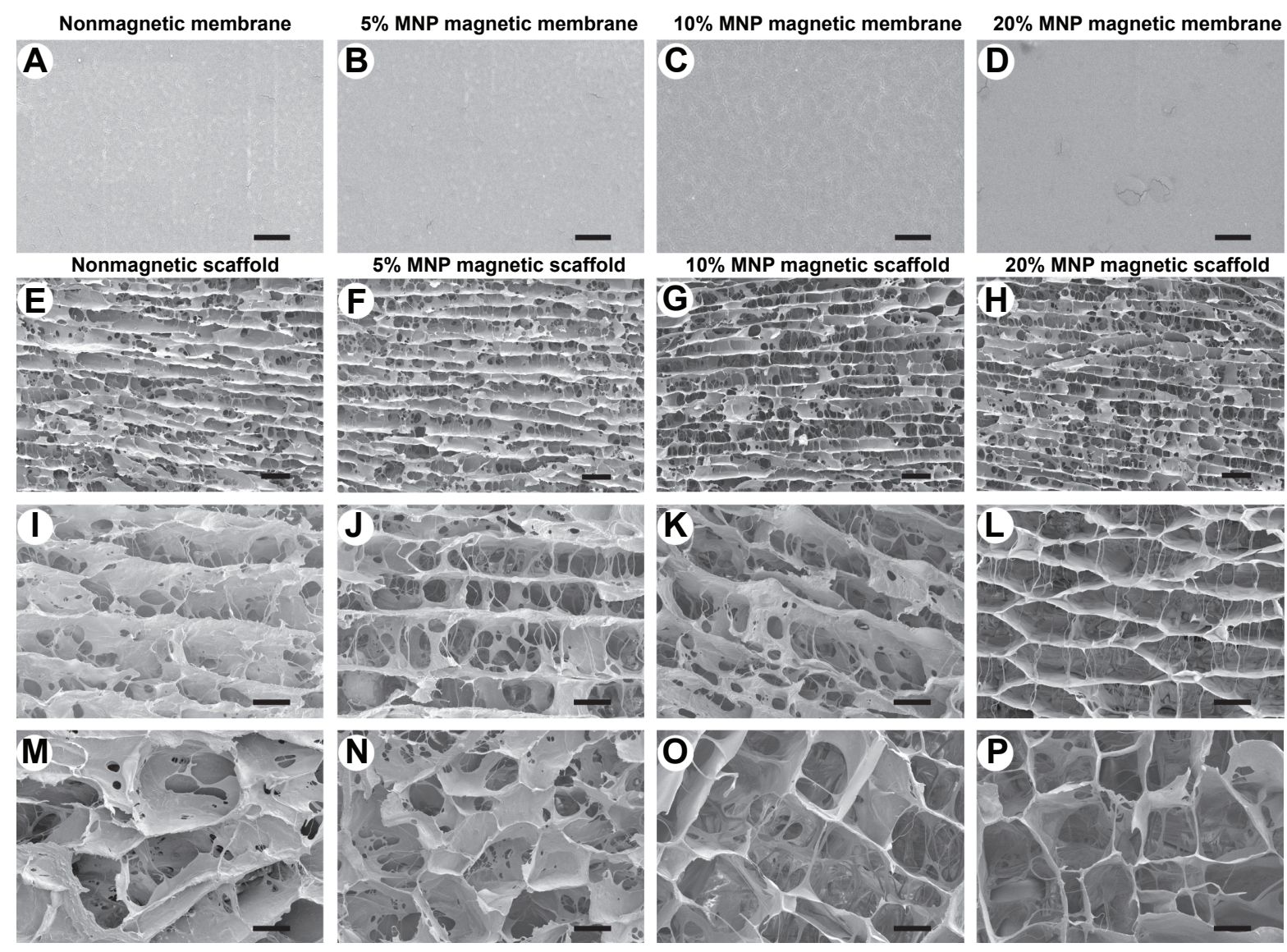

Figure 6 SEM images of the magnetic membranes and scaffolds.

Notes: (A-D) Representative images of nonmagnetic membrane, 5\% MNP magnetic membrane, 10\% MNP magnetic membrane, and 20\% MNP magnetic membrane are shown in the first row. (E-L) Representative images of nonmagnetic scaffold, $5 \%$ MNP magnetic scaffold, $10 \%$ MNP magnetic scaffold, and $20 \%$ MNP magnetic scaffold in a longitudinal section, showing the longitudinally oriented microchannels and interconnected porous structure. (M-P) Representative images of nonmagnetic scaffold, $5 \%$ MNP magnetic scaffold, 10\% MNP magnetic scaffold, and 20\% MNP magnetic scaffold in a transverse section, showing that the microchannels were arranged in a honeycomb-like pattern. Scale bars: (A-D) $1.5 \mu \mathrm{m},(\mathbf{E}-\mathbf{H}) 60 \mu \mathrm{m},(\mathbf{I}-\mathbf{P}) 30 \mu \mathrm{m}$.

Abbreviations: SEM, scanning electron microscopy; MNPs, magnetic nanoparticles.

scaffolds was variable, with $34.52 \pm 1.93 \mu \mathrm{m}$ in $5 \% \mathrm{MNP}$ magnetic scaffolds, $32.33 \pm 1.81 \mu \mathrm{m}$ in $10 \%$ MNP magnetic scaffolds, and $26.75 \pm 1.71 \mu \mathrm{m}$ in $20 \%$ MNP magnetic scaffolds, as well as $36.55 \pm 1.87 \mu \mathrm{m}$ in nonmagnetic scaffolds.

\section{Scaffold porosity}

The composition had an effect on the porosity of the magnetic scaffolds. Porosity was $78.48 \% \pm 0.89 \%$ in $20 \%$ MNP magnetic scaffolds, which was significantly lower than that in nonmagnetic scaffolds $(82.70 \% \pm 0.85 \%)$ and $5 \%(81.09 \% \pm 0.64 \%)$ and $10 \%(80.41 \% \pm 0.72 \%)$ MNP magnetic scaffolds $(P<0.05)$. The $10 \%$ MNP magnetic nanocomposites were used for the remainder of the experiments in this study, due to their good morphological features and magnetic performance.

\section{SC survival on magnetic nanocomposites under MFs}

The effect of MFs on the survival of SCs with or without $10 \%$ MNP magnetic nanocomposites was investigated by flow-cytometry. As shown in Figure 7, the apoptosis ratio of SCs cultured on magnetic membranes was $1.34 \% \pm 0.15 \%$ at $0.5 \mathrm{mT}, 1.66 \% \pm 0.18 \%$ at $1.0 \mathrm{mT}$, and $1.58 \% \pm 0.14 \%$ at $2.0 \mathrm{mT} 24$ hours after MF exposure, respectively, which was in a similar range to that on culture plates and nonmagnetic and magnetic membranes with no MF. When the MF was increased to $5.0 \mathrm{mT}$, the percentages of apoptotic cells cultured on nonmagnetic membranes and magnetic membranes significantly increased to $22.70 \% \pm 0.93 \%$ $(P<0.01)$ and $26.06 \% \pm 1.03 \%(P<0.01)$, respectively. Furthermore, when the MF was increased to $10.0 \mathrm{mT}$, the percentages of apoptotic cells cultured on nonmagnetic membranes and magnetic membranes increased significantly to $68.08 \% \pm 0.94 \%(P<0.01)$ and $76.92 \% \pm 0.84 \%$ $(P<0.01)$, respectively, indicating that higher MFs (5 and $10 \mathrm{mT}$ ) are detrimental to SCs seeded on magnetic or nonmagnetic substrates. Therefore, MF at $2.0 \mathrm{mT}$ was applied in the remaining experiments, as it led to nonsignificant cell apoptosis. 

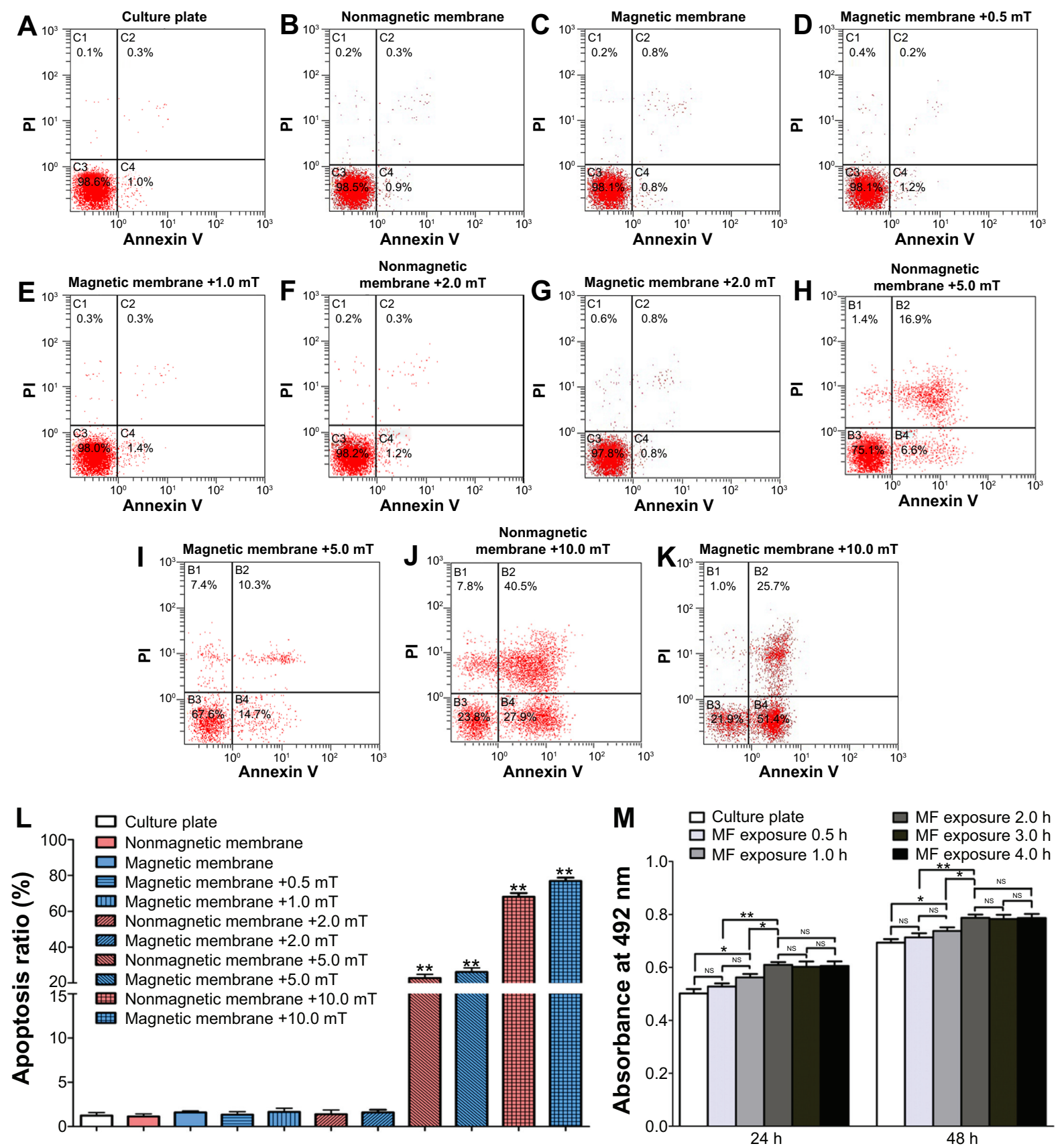

Figure 7 Apoptosis assay of SCs in each group by flow cytometry and CCK-8 assay.

Notes: (A) Culture-plate group; (B) nonmagnetic membrane group; (C) magnetic membrane group; (D) magnetic membrane + MF group (0.5 mT); (E) magnetic membrane + MF group (1.0 mT); (F) nonmagnetic membrane + MF group (2.0 mT); (G) magnetic membrane + MF group $(2.0 \mathrm{mT}) ;(\mathbf{H})$ nonmagnetic membrane + MF group $(5.0 \mathrm{mT})$; (I) magnetic membrane + MF group (5.0 mT); (J) nonmagnetic membrane + MF group (10.0 mT); (K) magnetic membrane + MF group (I0.0 mT). (L) The percentage of apoptotic cells in each group was obtained by averaging the results of five flow-cytometry assays for each group. (M) The CCK-8 values of viable cells in each group were obtained. All data are expressed as means \pm standard error of mean. $* P<0.05$; $* * P<0.01$, one-way ANOVA when compared with culture-plate group.

Abbreviations: SCs, Schwann cells; MF, magnetic field; CCK, Cell Counting Kit; ANOVA, analysis of variance; Pl, propidium iodide; h, hours; NS, not significant.

\section{CCK-8 assay}

Twenty-four hours after MF exposure, the CCK-8 value of SCs under 2 hours of MF stimulation was 1.15- and 1.08 -fold higher than that under 0.5 and 1 hour of MF stimulation, respectively; however, the CCK-8 values under 2, 3, and 4 hours of MF stimulation showed no significant difference (Figure 7M). Forty-eight hours after MF exposure, the CCK- 8 value of SCs under 2 hours of MF stimulation was 1.10- and 1.07-fold higher than that under 0.5 and 1 hour of MF stimulation, respectively; however, the CCK-8 values under 2, 3, and 4 hours of MF stimulation showed no significant difference (Figure 7M). 
Therefore, a 2-hour exposure time of MFs was applied in the remaining experiments.

\section{SC morphology on membranes and scaffolds under MFs}

Without MF exposure, SCs attached and expanded on the magnetic membranes 24 hours after seeding (Figure 8C), which was comparable to those cultured on nonmagnetic membranes (Figure 8B) and culture plates (Figure 8A), indicating that magnetic membranes were nontoxic to SCs.
Twenty-four hours after MF exposure (2.0 mT), SCs exhibited a spindle shape and attached well on both nonmagnetic (Figure 8E) and magnetic membranes (Figure 8F). In particular, cell division was observed on the $10 \%$ magnetic membranes under MF (2.0 mT, Figure 8G). In contrast, cells were almost round, with damaged membranes and cell debris under MFs at $5.0 \mathrm{mT}$ or $10.0 \mathrm{mT}$ after 24 hours' exposure (Figure 8, H and I). The SEM results on membranes were consistent with the flow-cytometry assay. The dendritic length of SCs on culture plates and nonmagnetic and magnetic
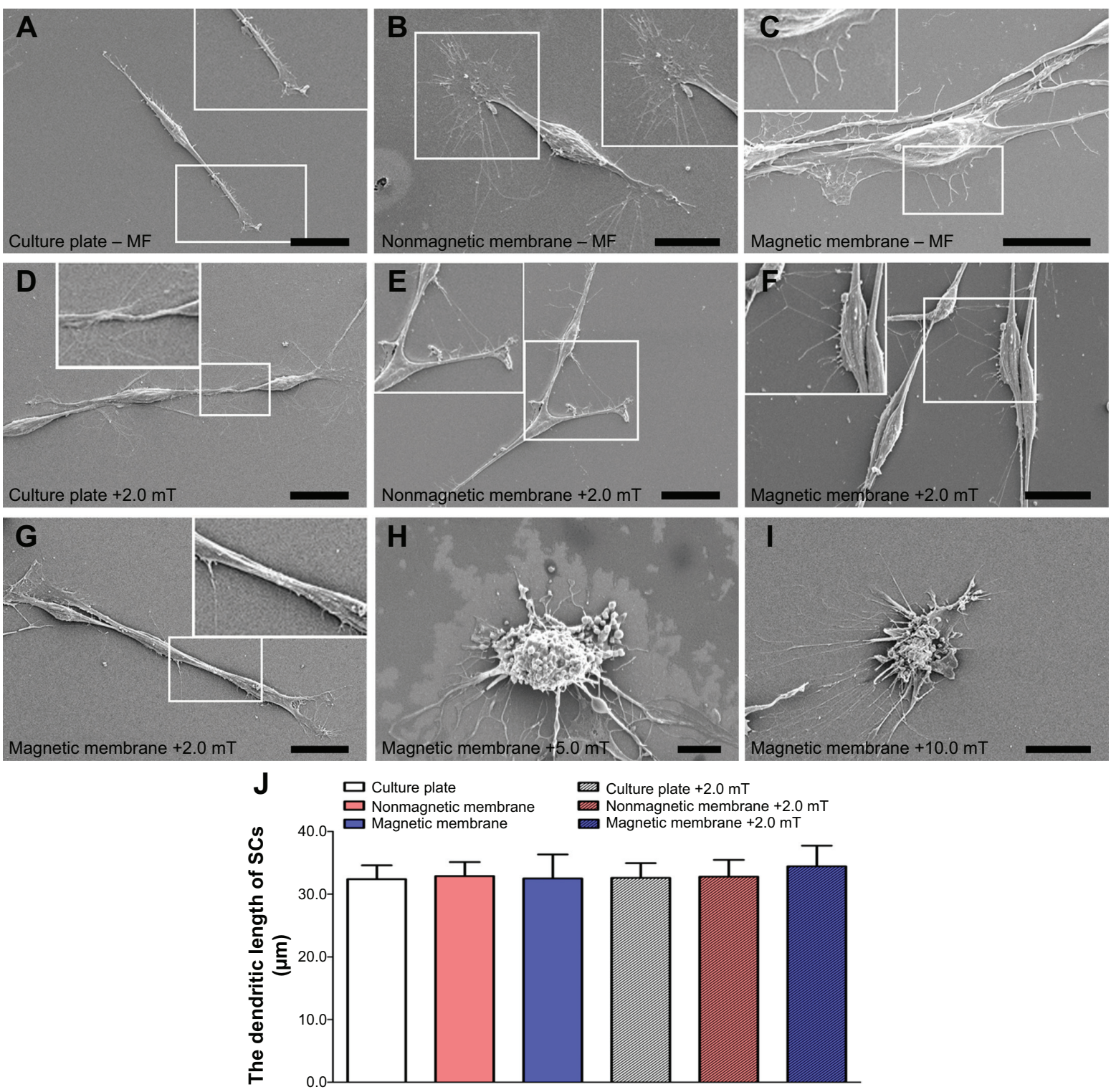

Figure 8 Representative SEM photomicrographs of SCs in each group at 24 hours after seeding.

Notes: (A) Culture-plate group; (B) nonmagnetic membrane group; (C) magnetic membrane group; (D) culture plate + MF group (2.0 mT); (E) nonmagnetic membrane + MF group (2.0 mT); (F) magnetic membrane + MF group (2.0 mT); (G) dividing phase in magnetic membrane + MF group (2.0 mT); (H) magnetic membrane + MF group $(5.0 \mathrm{mT})$; (I) magnetic membrane + MF group (10.0 mT). (J) The dendritic length of SCs in each group was measured (n=20). Scale bar: (A, D, E, G) $20 \mu \mathrm{m},(\mathbf{B}, \mathbf{F}, \mathbf{I}) \mathbf{I} 5 \mu \mathrm{m}$, (C) $10 \mu \mathrm{m},(\mathbf{H}) 5 \mu \mathrm{m}$.

Abbreviations: SCs, Schwann cells; MF, magnetic field. 
membranes with MF exposure $(2.0 \mathrm{mT})$ was $32.58 \pm 2.35 \mu \mathrm{m}$, $32.77 \pm 2.69 \mu \mathrm{m}$, and $34.41 \pm 3.30 \mu \mathrm{m}$, respectively, and without MF exposure was $32.37 \pm 2.20 \mu \mathrm{m}, 32.86 \pm 2.24 \mu \mathrm{m}$, and $32.49 \pm 3.82 \mu \mathrm{m}$, respectively (Figure $8 \mathrm{~J}$ ), indicating that magnetic membranes via MF had no significant effect on the dendritic length of SCs.

SCs cocultivated with scaffolds were also observed (Figure 10, A-D). Without MF exposure, SCs attached, adhered, and expanded on the nonmagnetic and magnetic scaffolds 24 hours after coculture, confirming that the 10\% MNP magnetic scaffold was nontoxic to SCs. Twenty-four hours after MF exposure (2.0 mT), SCs exhibited a spindle shape and adhered well on both nonmagnetic and magnetic scaffolds.

\section{SC viability on magnetic nanocomposites under MFs}

The cell viability and number of SCs on magnetic membranes or scaffolds were investigated with or without MF. The EdU-labeling index was used to quantify cell proliferation on membranes. We first examined the effect of MF on cell proliferation. It was found that MF had no significant effect on the EdU-labeling index on culture plates or nonmagnetic membranes. We next examined whether magnetic membranes via MF could influence the effect of cell proliferation. When SCs were cultured on magnetic membranes, the EdU-labeling index of SCs was 1.73-fold higher than that without MF (Figure 9, C, F, and G), indicating that magnetic membranes via $\mathrm{MF}$ are capable of promoting cell proliferation. Without MF exposure, the PrestoBlue value of SCs was in a similar range on culture plates and nonmagnetic and magnetic membranes after 24 hours. Under MF exposure, the PrestoBlue value of SCs cultured on magnetic membranes was 1.53-fold higher than that without MF after 24 hours' culture. In addition, the PrestoBlue value of SCs on culture plates and nonmagnetic membranes showed no significant difference compared to that without MF after 24 hours' culture (Figure 9H).

The survival of SCs in magnetic scaffolds with or without MF was then investigated by Live/Dead staining. The representative images of Live/Dead assays are shown in Figure 10, E-P. Without MF exposure, the percentage of live cells was in a similar range on nonmagnetic and magnetic scaffolds after 24 hours. Under MF exposure, the percentage of live cells was $78.97 \% \pm 1.03 \%$ on nonmagnetic scaffolds and $84.84 \% \pm 1.31 \%$ on magnetic scaffolds after 24 hours' exposure (Figure 10Q). In addition, the percentage of live cells on magnetic scaffolds under MF exposure increased by 1.05-fold at day 3 and 1.09-fold at day 5 compared to that at day 1 (Figure 10Q).
The viability of SCs in magnetic scaffolds was further examined with or without MF by PrestoBlue assay. Without MF exposure, the PrestoBlue value of SCs was in a similar range on culture plates and nonmagnetic and magnetic scaffolds after 24 hours (Figure 10R). Under MF exposure, the PrestoBlue value of SC-cocultivated magnetic scaffolds was 1.58 -fold higher than that without MF after 24 hours' culture.

\section{Expression of regeneration-related genes}

The effect of MFs on expression of BDNF, GDNF, NT-3, and $V E G F$ of SCs seeded with membranes and scaffolds was examined by RT-PCR 12, 24, and 36 hours after MF exposure. The mRNA levels of $B D N F, G D N F, N T-3$, and $V E G F$ were in a similar range as $\mathrm{SCs}$ cultured in groups without $\mathrm{MF}$ exposure at 12, 24, and 36 hours, respectively. Under MF exposure, the $B D N F$ mRNA level cultured on magnetic membranes and magnetic scaffolds at 12 hours was 1.31- and 1.64-fold higher than that without MF exposure, respectively, at 24 hours was 1.76- and 2.07-fold higher than that without MF exposure, respectively, and at 36 hours was 1.50- and 1.72-fold higher than that without MF exposure, respectively (Figure 11A). The GDNF mRNA level cultured on magnetic membranes and magnetic scaffolds at 12 hours was 1.50- and 1.79-fold higher than that without MF exposure, respectively, at 24 hours was 1.91- and 2.29-fold higher than that without MF exposure, respectively, and at 36 hours was 1.58- and 1.92-fold higher than that without MF exposure, respectively (Figure 11B). The NT-3 mRNA level cultured on magnetic membranes and magnetic scaffolds at 12 hours was 1.18- and 1.26-fold higher than that without MF exposure, respectively, at 24 hours was 1.34- and 1.55-fold higher than that without MF exposure, respectively, and at 36 hours was 1.24- and 1.38-fold higher than that without MF exposure, respectively (Figure 11C). The VEGF mRNA level cultured on magnetic membranes and magnetic scaffolds at 12 hours was 1.44- and 1.83-fold higher than that without MF exposure, respectively, at 24 hours was 2.02- and 2.51-fold higher than that without MF exposure, respectively, and at 36 hours was 1.69- and 2.07-fold higher than that without MF exposure, respectively (Figure 11D).

\section{Secretion of regeneration-related proteins}

The effect of MFs on the secretion of BDNF, GDNF, NT-3, and VEGF of SCs seeded with membranes and scaffolds was examined 12, 24, and 36 hours after MF exposure. The secretion of BDNF, GDNF, NT-3, and VEGF were in a similar range in SCs cultured in groups without MF exposure at 12, 24, and 36 hours, respectively. Under MF exposure, 

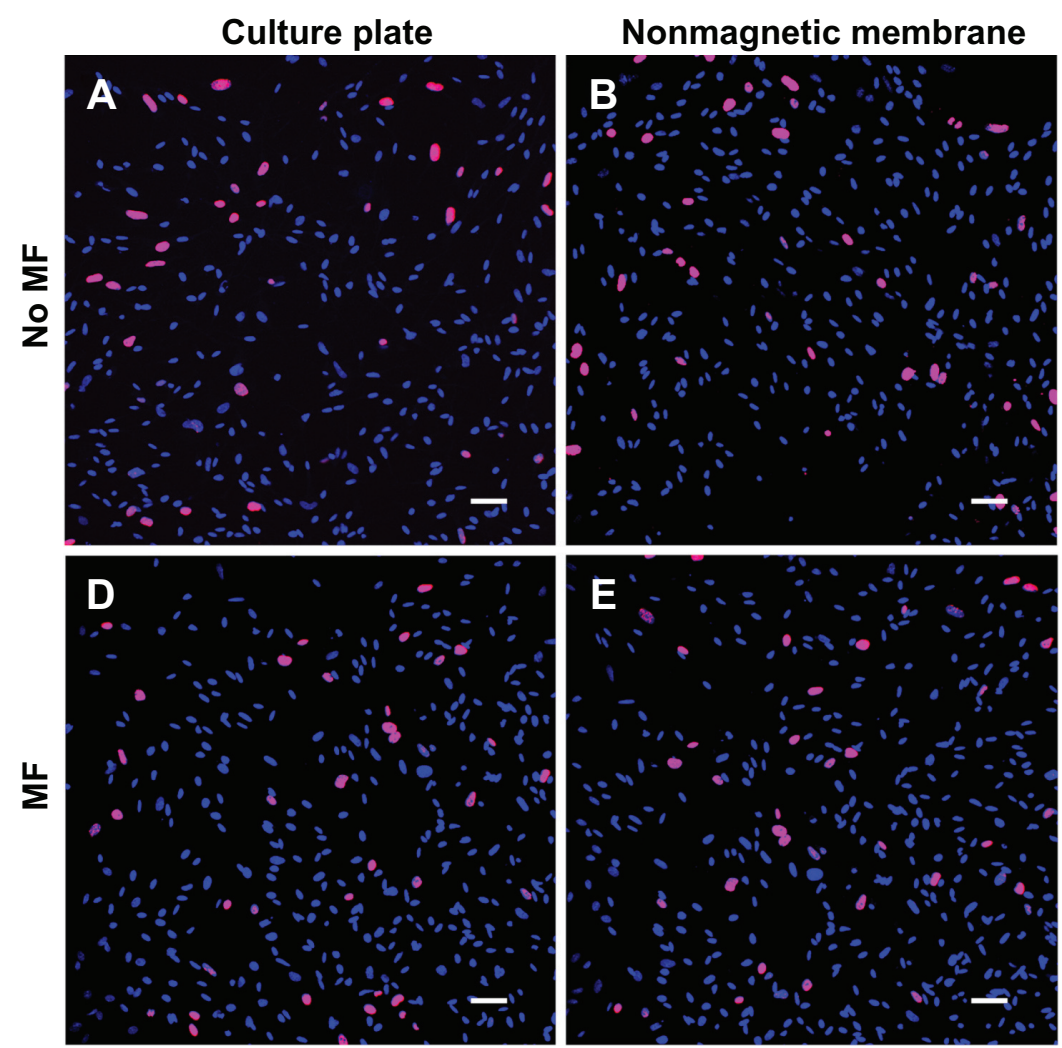

Magnetic membrane
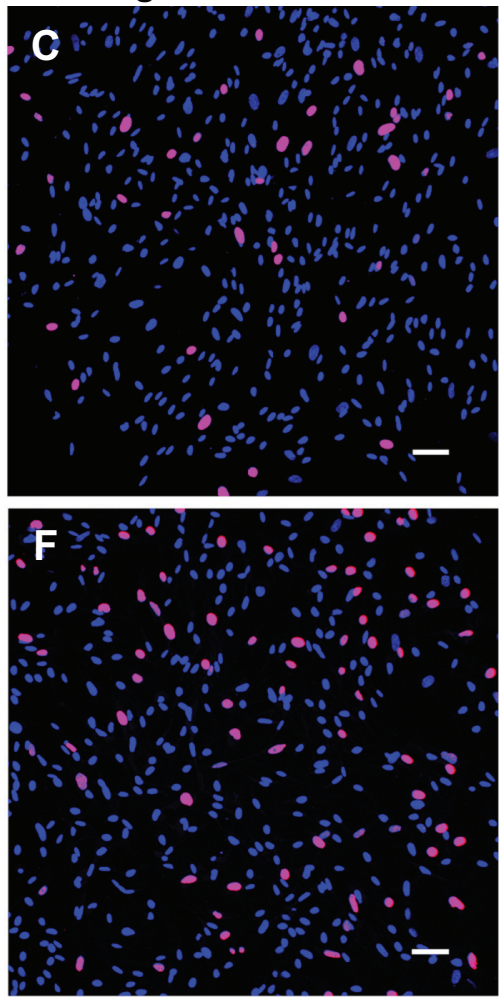
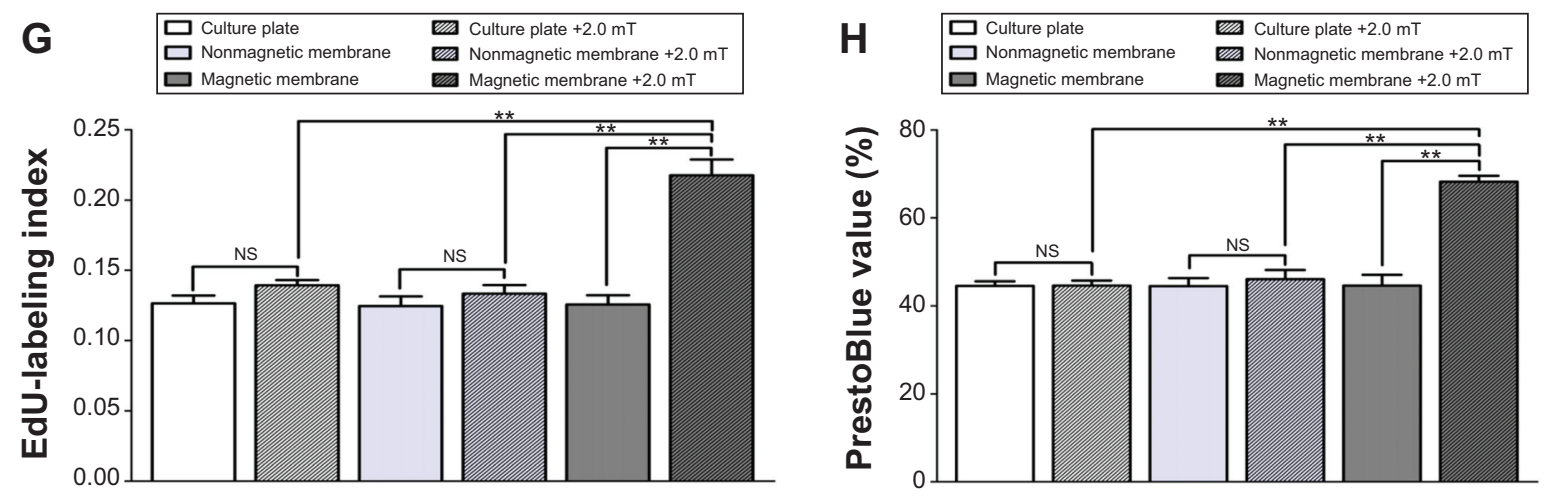

Figure $9 \mathrm{EdU}$ staining and PrestoBlue assay in magnetic membranes at 24 hours after seeding.

Notes: (A) culture plate group; (B) nonmagnetic membrane group; (C) magnetic membrane group; (D) culture plate + MF group (2.0 mT); (E) nonmagnetic membrane + MF group (2.0 mT); (F) magnetic membrane + MF group $(2.0 \mathrm{mT})$. The EdU-labeling index $(\mathbf{G})$ and PrestoBlue assay values $(\mathbf{H})$ in each group were obtained by averaging the results of five samples for each group. Scale bar: (A-F) $50 \mu \mathrm{m}$ (magnification $20 \times$ ). All data are expressed as means \pm standard error of mean. $* * P<0.01$.

Abbreviations: EdU, 5-ethynyl-2-deoxyuridine; MF, magnetic field; NS, not significant.

the secretion of BDNF cultured on magnetic membranes and magnetic scaffolds at 12 hours was 1.38 - and 1.54-fold higher than that without MF exposure, respectively, at 24 hours was 1.75- and 2.11-fold higher than that without MF exposure, respectively, and at 36 hours was 1.44- and 1.77fold higher than that without MF exposure, respectively (Figure 12A). The secretion of GDNF cultured on magnetic membranes and magnetic scaffolds at 12 hours was 1.50- and 1.88-fold higher than that without MF exposure, respectively, at 24 hours was 2.22 - and 2.83 -fold higher than that without MF exposure, respectively, and at 36 hours was
1.55- and 2.04-fold higher than that without MF exposure, respectively (Figure 12B). The secretion of NT-3 cultured on magnetic membranes and magnetic scaffolds at 12 hours was 1.64- and 2.40-fold higher than that without MF exposure, respectively, at 24 hours was 2.81- and 4.52-fold higher than that without MF exposure, respectively, and at 36 hours was 2.00- and 2.90-fold higher than that without MF exposure, respectively (Figure 12C). The secretion of VEGF cultured on magnetic membranes and magnetic scaffolds at 12 hours was 1.23- and 1.46-fold higher than that without MF exposure, respectively, at 24 hours was 1.65 - and 2.45-fold 

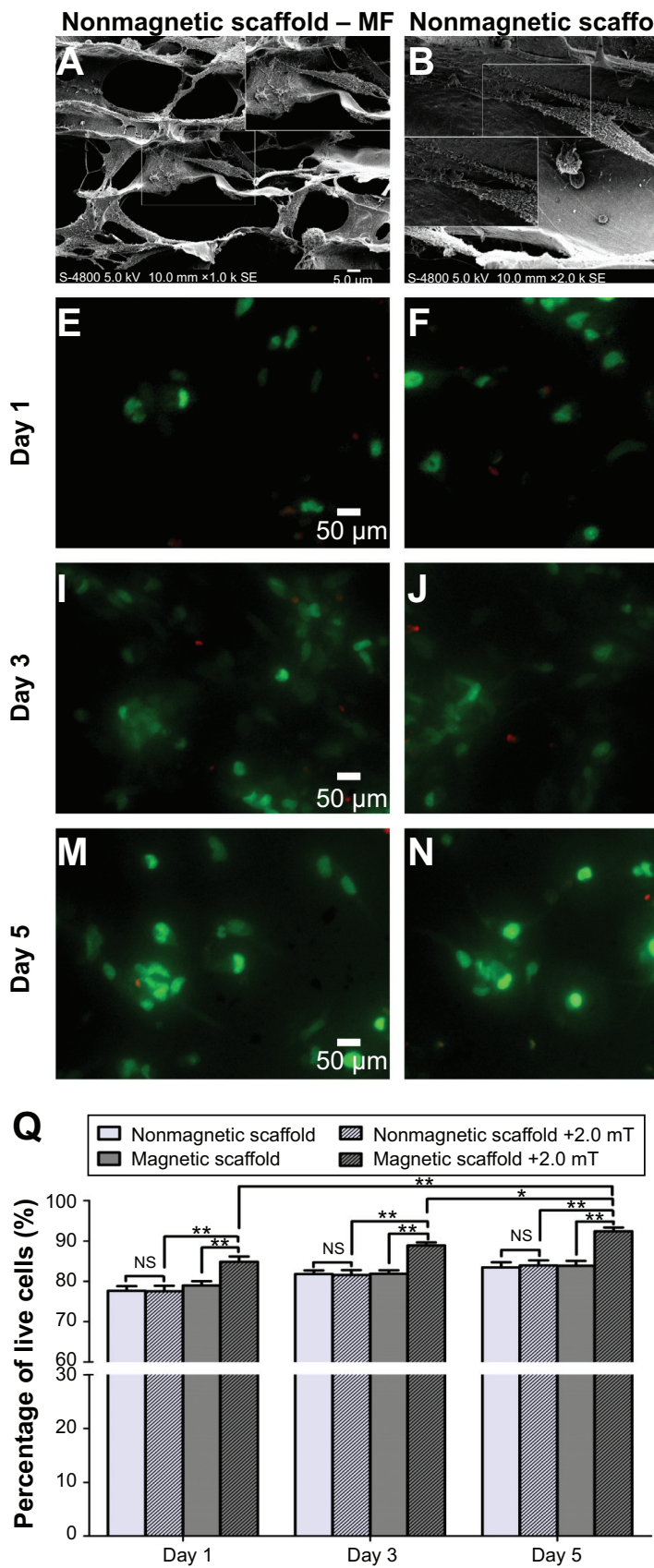
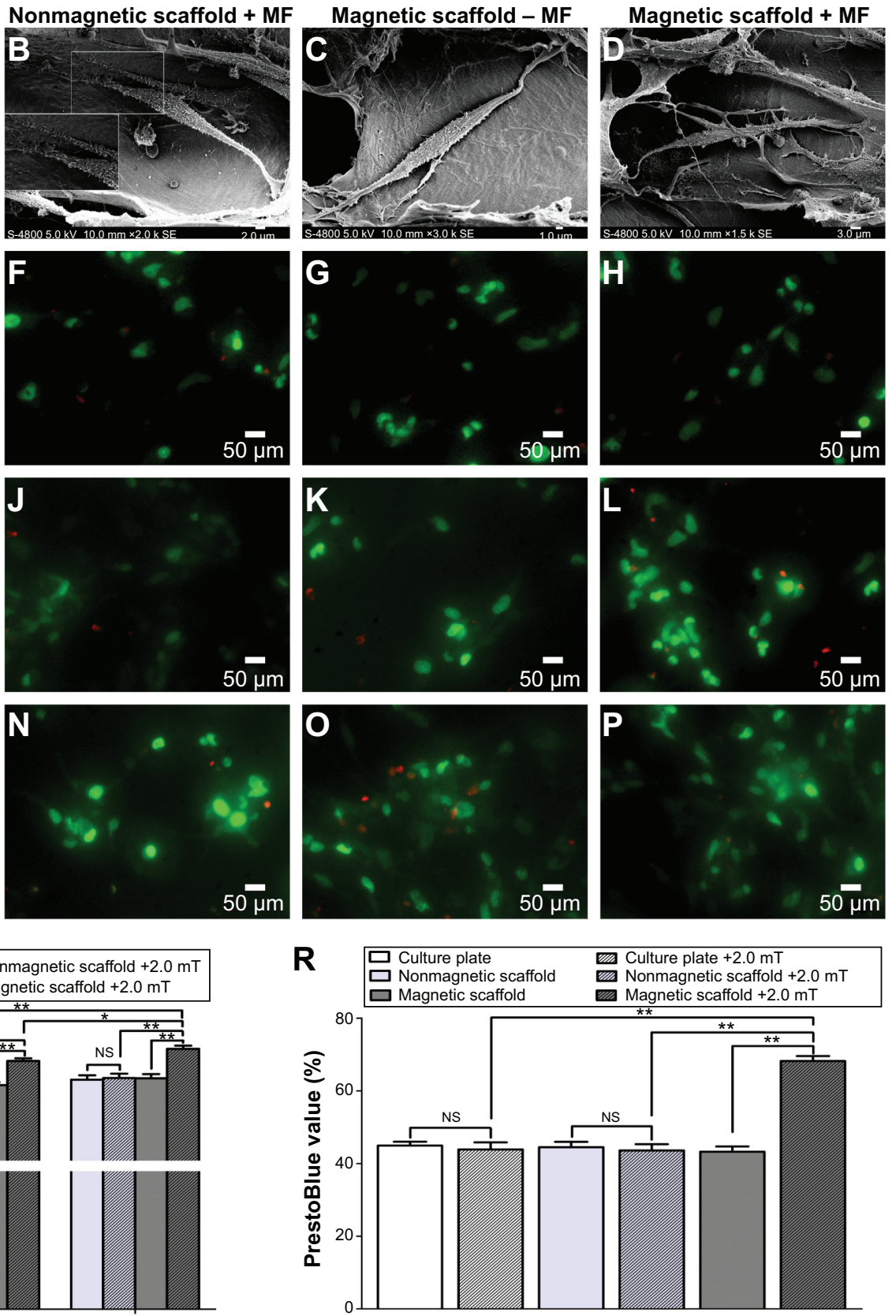

Figure 10 SEM and Live/Dead staining of the SCs cocultivated with scaffold in each group.

Notes: (A-D) Representative images of SEM at 24 hours after seeding, (E-H) Live/Dead staining at Day I, (I-L) Day 3, and (M-P) Day 5 in the nonmagnetic scaffold group, nonmagnetic scaffold + MF group (2.0 mT), magnetic scaffold group, and magnetic scaffold + MF group (2.0 mT). The percentage of living SCs (Q) and PrestoBlue assay values $(\mathbf{R})$ in each group were obtained by averaging the results of five samples for each group. Scale bars: (E-P) $50 \mu \mathrm{m}$ (magnification $60 \times)$. All data are expressed as means \pm standard error of mean. $* P<0.05 ; * * P<0.01$.

Abbreviations: SEM, scanning electron microscopy; SCs, Schwann cells; MF, magnetic field; NS, not significant.

higher than that without MF exposure, respectively, and at 36 hours was 1.30 - and 1.79-fold higher than that without MF exposure, respectively (Figure 12D).

\section{Discussion}

The present study first fabricated and characterized novel magnetically responsive nanocomposites, and then found that magnetic nanocomposites via MFs were capable of enhancing the biological properties of SCs. These findings open up possibilities of promoting nerve regeneration in these magnetically responsive nanocomposites through enhanced viability and neurotrophins secretion of SCs.

MNPs are superparamagnetic at room temperature, which makes them attractive in applications in biomedical fields, 
A

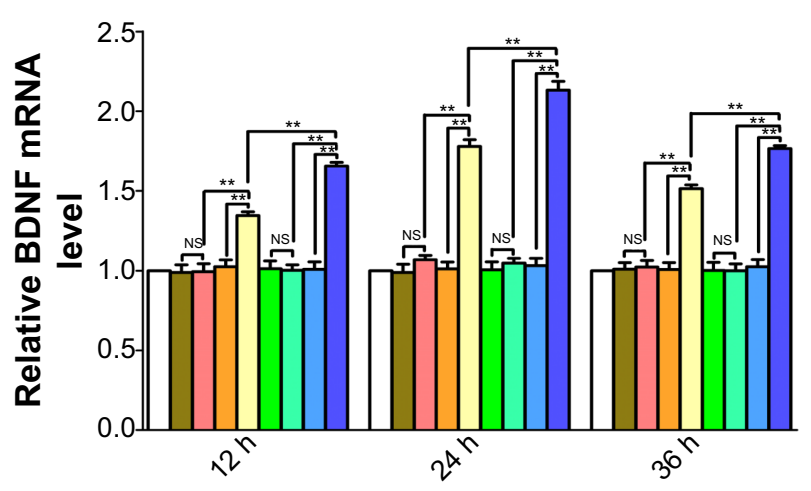

C

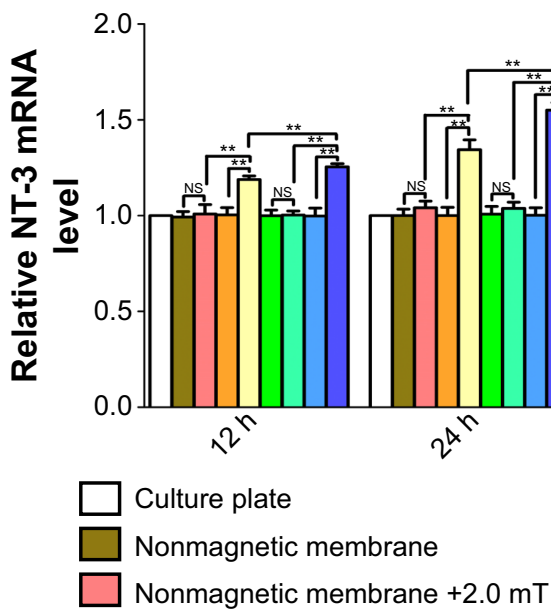

$3^{60}$
B

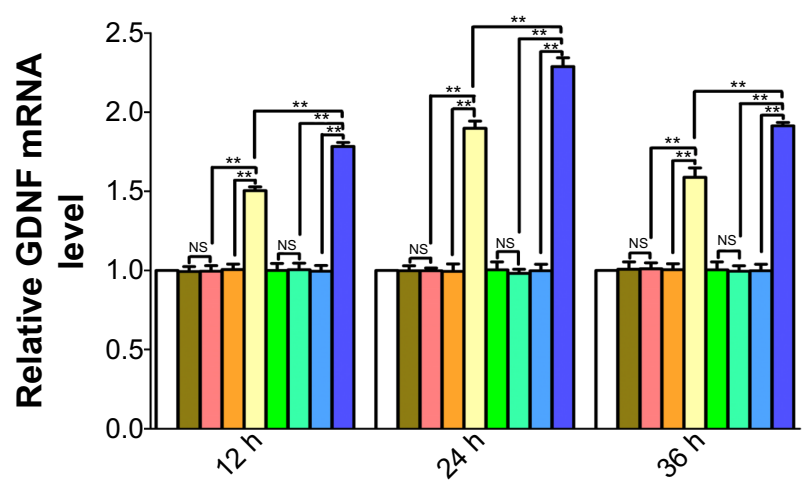

D
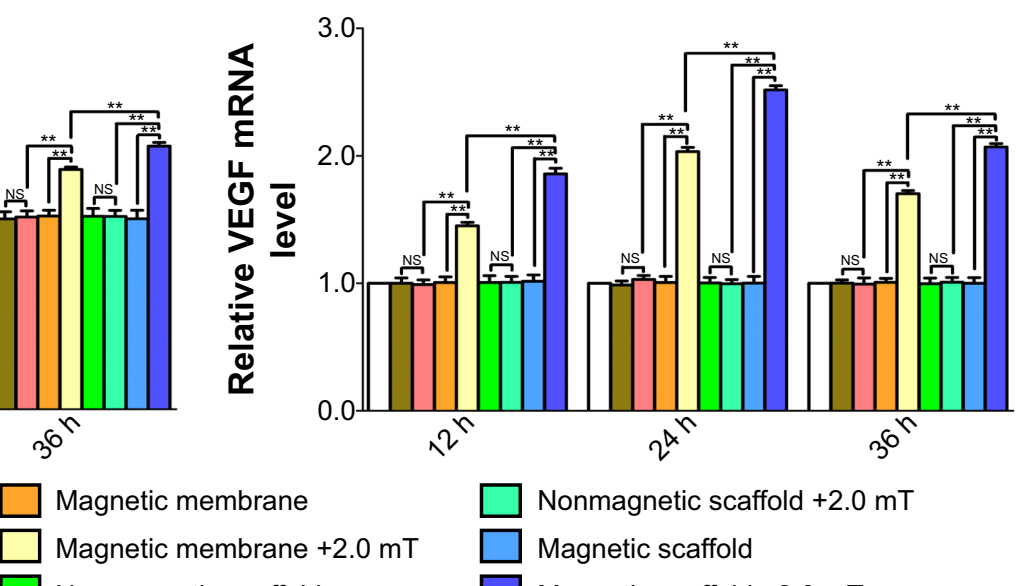

$\square$ Nonmagnetic scaffold $\square$ Nonmagnetic scaffold $+2.0 \mathrm{mT}$

Magnetic scaffold

Magnetic scaffold $+2.0 \mathrm{mT}$

Figure II mRNA levels of BDNF, GDNF, NT-3, and VEGF in each group with or without MF at 12, 24, and 36 hours after MF.

Notes: mRNA levels of (A) BDNF, (B) GDNF, (C) NT-3, and (D) VEGF were determined for the culture-plate group, nonmagnetic membrane group, nonmagnetic membrane + MF group (2.0 mT), magnetic membrane group, magnetic membrane + MF group $(2.0 \mathrm{mT})$, nonmagnetic scaffold group, nonmagnetic scaffold + MF group (2.0 mT), magnetic scaffold group, and magnetic scaffold + MF group $(2.0 \mathrm{mT})$. Each test was repeated three times. The ratio of mRNA levels of the SC nonmagnetic membrane group, nonmagnetic membrane + MF group $(2.0 \mathrm{mT})$, magnetic membrane group, magnetic membrane + MF group $(2.0 \mathrm{mT})$, nonmagnetic scaffold group, nonmagnetic scaffold + MF group $(2.0 \mathrm{mT})$, magnetic scaffold group, and magnetic scaffold + MF group $(2.0 \mathrm{mT})$ to the culture plate group are shown. All data are expressed as means \pm standard error of mean. $* * P<0.01$.

Abbreviations: mRNA, messenger ribonucleic acid; BDNF, brain-derived neurotrophic factor; GDNF, glial cell-derived neurotrophic factor; NT-3, neurotrophin 3; VEGF, vascular endothelial growth factor; MF, magnetic field; SC, Schwann cell; NS, not significant; h, hours.

such as hyperthermia therapy, drug delivery, and magnetic resonance imaging. ${ }^{22-24} \mathrm{~A}$ recent study has reported that MNP-bound BDNF can transmigrate across the blood brain barrier using an in vitro blood brain barrier model and subsequently exert the role of BDNF in neuroprotection, which represents an extensive application of MNPs with additional molecular factors. ${ }^{25}$ In addition, chitosan and glycerophosphate are biodegradable, mucoadhesive, and nontoxic polymers, which show good biocompatibility for neuron survival and adherence. ${ }^{26}$ Therefore, the magnetically responsive nanocomposites in the present study were made of chitosan, glycerophosphate, and MNPs. The FTIR and XRD results indicated that the structure of MNPs in the magnetic nanocomposites was well maintained during stirring and lyophilization, and this was very critical for keeping their magnetic behavior. Furthermore, the magnetic nanocomposites exhibited superparamagnetism at room temperature, which made them work like a local MF amplifier. Their intrinsic magnetization could be triggered by an applied MF, and continuous interaction of the nanocomposite with the external field can be activated under the effect of magnetically responsive MNPs.

Microstructural characteristics are crucial for tissueengineering applications of biomaterials. In this study, the magnetic scaffolds made of $5 \%$ and $10 \%$ MNP magnetic nanocomposites showed porosities close to $85 \%$, which has been shown to be necessary to allow the migration of regenerating axons in ample space. ${ }^{27}$ In contrast, the porosity of $20 \%$ MNP magnetic scaffolds had porosity of less than $80 \%$, which might be attributable to the cross-linking role 

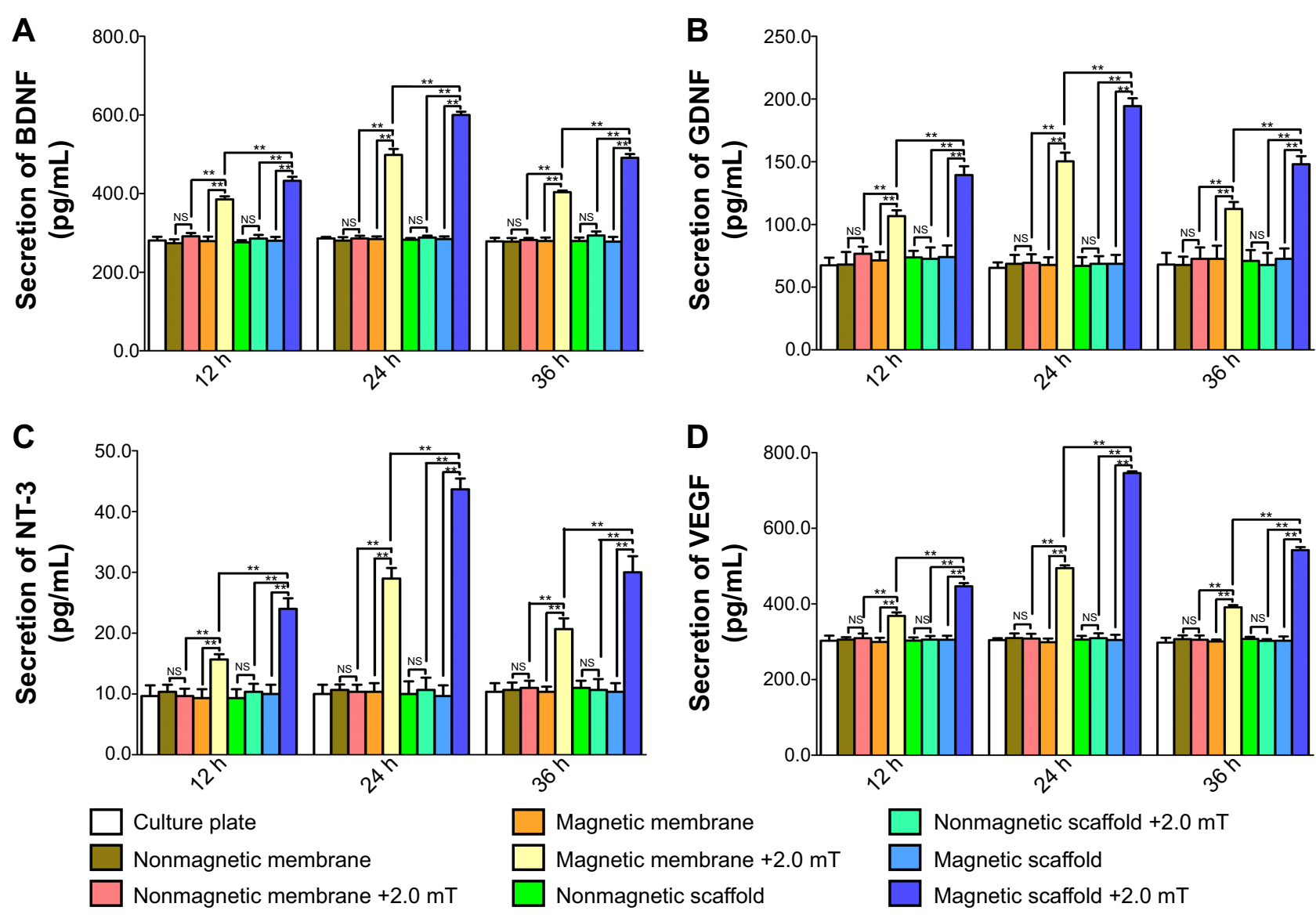

$\square$ Nonmagnetic scaffold $+2.0 \mathrm{mT}$

$\square$ Magnetic scaffold

$\square$ Magnetic scaffold $+2.0 \mathrm{mT}$

Figure 12 Secretion of BDNF, GDNF, NT-3, and VEGF in each group with or without MF at 12, 24, and 36 hours after MF.

Notes: Secretion levels of (A) BDNF, (B) GDNF, (C) NT-3, and (D) VEGF were determined for the culture-plate group, nonmagnetic membrane group, nonmagnetic membrane + MF group (2.0 mT), magnetic membrane group, magnetic membrane + MF group ( $2.0 \mathrm{mT})$, nonmagnetic scaffold group, nonmagnetic scaffold + MF group ( 2.0 $\mathrm{mT})$, magnetic scaffold group, and magnetic scaffold + MF group $(2.0 \mathrm{mT})$. Each test was repeated three times. Protein-level ratios of the SC nonmagnetic membrane group, nonmagnetic membrane + MF group $(2.0 \mathrm{mT})$, magnetic membrane group, magnetic membrane + MF group ( $2.0 \mathrm{mT})$, nonmagnetic scaffold group, nonmagnetic scaffold + MF group ( $2.0 \mathrm{mT})$, magnetic scaffold group, and magnetic scaffold + MF group $(2.0 \mathrm{mT})$ to the culture-plate group are shown. All data are expressed as means \pm standard error of mean. $* * P<0.01$.

Abbreviations: BDNF, brain-derived neurotrophic factor; GDNF, glial cell-derived neurotrophic factor; NT-3, neurotrophin 3; VEGF, vascular endothelial growth factor; MF, magnetic field; SC, Schwann cell; NS, not significant; h, hours.

of MNPs in the chitosan-glycerophosphate polymer. ${ }^{28}$ The cross-linking property of MNPs in scaffolds was realized by reducing the biopolymer-chain distance, thus resulting in the possibilities of more sealed pores, less pore interconnection, and lower porosity with more MNPs. In addition, the cross-sectional diameter of microchannels in the 5\% and 10\% MNP magnetic scaffolds were approximately $30 \mu \mathrm{m}$, which has been shown to be most effective for promoting orientated neurite outgrowth, ${ }^{29}$ since it is small enough to physically align and restrict the orientation of regenerating axons, as well as large enough to allow for vascularization and the infiltration of cells that support regeneration. ${ }^{30-32}$

It has been shown that the intensity of MFs is crucial for their effect on biological properties of different cell types, including rat bone mesenchymal stromal cells, PC12 cells and neuroblastoma cells. ${ }^{9,19,20}$ In the present study, we first screened the optimal intensity of MFs for SCs. It was found that $\mathrm{MF}$ at $2 \mathrm{mT}$ was beneficial for the attachment and proliferation of SCs in membranes and scaffolds. When MFs increased to high magnetic gradients $(5 \mathrm{mT}$ and $10 \mathrm{mT}$ ), the apoptosis ratio of SCs significantly increased in contrast to the MF at $2 \mathrm{mT}$, indicating that MFs at higher magnetic intensities lead to significant cell apoptosis and are detrimental to SC viability. It has been shown that $\mathrm{MF}$ at $0.016 \mathrm{mT}$ had no detectable effect on neurite outgrowth in PC12 cells, while a high $\mathrm{MF}$ at $1.37 \mathrm{mT}$ significantly promoted neurite outgrowth. ${ }^{19}$ In addition, MF at $5 \mathrm{mT}$ has been found to facilitate neuronal differentiation of rat bone mesenchymal stromal cells. ${ }^{9}$ All these findings imply that the sensitivity of different cell types to MFs is distinct. In addition, 2 hours of MF exposure $(2.0 \mathrm{mT})$ was applied in the present 
study, for this exposure time showed higher CCK-8 value of SCs than that under 0.5 and 1 hour, and no significant difference compared with that under 3 and 4 hours of MF exposure. In a previous study, 20 minutes and 5 days of MF exposure was applied to regulate the biological properties of endothelial cells. ${ }^{12}$ However, studies comparing the efficacy of MFs with different exposure durations on SCs through magnetic nanocomposites have been lacking, and these may be important for in vivo and clinical applications of MFs in nerve-injury repair. Therefore, optimal parameters of MFs through magnetic nanocomposites for SCs need to be chosen before in vivo and clinical applications of MFs in nerve-injury repair.

We then investigated whether magnetic nanocomposites via MFs are capable of regulating SC biological properties. A significantly greater cell viability and higher EdU-labeling index were found in SCs cultured on magnetic membranes with MF than those without MF, suggesting that MF and magnetic materials worked synergistically to promote SC proliferation. In this study, the effect of MF and magnetic nanocomposites on SCs cocultivated within scaffolds was also investigated. It has been shown that a three-dimensional highly porous scaffold provides surfaces on which cells adhere, thrive, multiply, and generate the extracellular matrix of structural and functional proteins and saccharides that make up living tissue. ${ }^{33}$ The SEM results showed that most of the SCs adhered well, and stretched out bipolar or multipolar processes with or without MFs, suggesting that magnetic scaffolds are capable of supporting SC adhesion. In addition, a significantly higher cell viability and higher percentage of live cells were found in SCs within magnetic scaffolds with MFs than those without MFs, suggesting that a magnetically responsive microenvironment inside threedimensional scaffolds is capable of enhancing cell viability and survival. All these findings highlight the possibility of combining MFs and SC-seeded magnetic scaffolds to enhance nerve regeneration in vivo.

SCs produce many neurotrophic factors, ${ }^{34,35}$ which are of great importance for promoting peripheral regeneration. ${ }^{36-38}$ In this study, the expression and secretion of BDNF, GDNF, NT-3, and VEGF was detected. BDNF has been reported to improve survival and outgrowth of motor neurons in both peripheral nerve and spinal cord injuries. ${ }^{39}$ GDNF is a neurotrophic factor that is critical for the growth, survival, and differentiation of motor neurons and central noradrenergic neurons. ${ }^{40} \mathrm{NT}-3$ is a major support protein for SCs, and loss of NT-3 results in degradation of SC efficiencies. ${ }^{41}$ In addition, VEGF is an effective protein that is able to promote axonal outgrowth and SC proliferation, and moreover, to stimulate SC infiltration and neovascularization after sciatic nerve transections. ${ }^{42,43}$ In the present study, magnetic membranes via MFs were capable of increasing the expression and secretion of BDNF, GDNF, NT-3, and VEGF in SCs, which was further enhanced when SCs were seeded within scaffolds, and moreover, the gene expression and protein secretion of these neurotrophic factors through a time-course assay were reversible, which has shown that high prolonged expression of these factors is not necessarily positive in peripheral nerve repair. ${ }^{44}$ All these findings indicated that magnetic nanocomposites via MFs are capable of regulating the expression and secretion of multiple NTs in a synergistic way in SCs.

Although the mechanism by which magnetic nanocomposites via MFs regulate the functions of SCs is unclear, a few possible causes could be postulated. First, because MNPs became an intrinsic component in the nanocomposites, they can be activated by applied MFs to create enormous miniature magnetic forces, and then trigger cell signaling via receptors, including channels and integrins on plasma membranes, and subsequently lead to activation of regeneration-related neurotrophic factors. Alternatively, it is possible that the magnetic nanocomposite undergoes a shape change by an external MF, creating mechanical forces that exert a mechanical effect on SCs. Further studies are needed to investigate the mechanism underneath the regulated effect on SCs in response to magnetic nanocomposites via MFs. In the present study, we incorporated MNPs in a chitosan-glycerophosphate composite, expecting to exert in the nanomaterial a novel magnetically responsive function. The benefit of incorporation of MNPs in nanocomposites is that MNPs become constitutional components, which through an applied MF continually stimulate SC proliferation and production of neurotrophic factors. Therefore, a combined application of MNP-functionalized nanocomposites and SCs is expected to provide a tunable regenerative microenvironment, which may be instructive for nerve regeneration and functional recovery after nerve injury and repair.

\section{Conclusion}

The present study describes the positive effect of magnetic nanocomposites and MFs on SC proliferation and biological properties in vitro. The magnetic nanocomposites in the present study were constructed from a chitosan-glycerophosphate polymer incorporated with magnetically responsive NPs. The magnetic nanocomposites hold the crystalline structure 
of MNPs, have modest magnetization, and possess a tunable microenvironment, making them appropriate for cellular support and cultivation in both membrane and scaffold environments. The magnetic nanocomposites and MFs work synergistically to regulate the biological properties of SCs and regulate the expression and secretion of BDNF, GDNF, NT-3, and VEGF in SCs. These findings open up possibilities of enhancing nerve regeneration in magnetically responsive scaffolds through enhanced viability and production of biologic factors in SCs via applied MFs.

\section{Acknowledgments}

This work was supported by grants from the National Natural Science Foundation of China (81201389 and 30973052), the National Basic Research Program of China (973 Program No 2014CB542206), and the Program for Changjiang Scholar and Innovative Research Team in University (IRT1053). We thank technicians Mr Jintao Hu, Ms Lifeng Lan, Ms Jing Fan, Mr Haifeng Zhang, Mr Yongqiang Li, and Ms Ying Hao for their excellent technical assistance.

\section{Disclosure}

The authors report no conflicts of interest in this work.

\section{References}

1. Fawcett JW, Keynes RJ. Peripheral nerve regeneration. Annu Rev Neurosci. 1990;13:43-60.

2. Chen ZL, Yu WM, Strickland S. Peripheral regeneration. Annu Rev Neurosci. 2007;30:209-233.

3. Zhang YG, Sheng QS, Qi FY, et al. Schwann cell-seeded scaffold with longitudinally oriented micro-channels for reconstruction of sciatic nerve in rats. J Mater Sci Mater Med. 2013;24(7):1767-1780.

4. Novikova LN, Pettersson J, Brohlin M, Wiberg M, Novikov LN. Biodegradable poly-beta-hydroxybutyrate scaffold seeded with Schwann cells to promote spinal cord repair. Biomaterials. 2008;29(9):1198-1206.

5. Schlosshauer B, Muller E, Schröder B, Planck H, Müller HW. Rat Schwann cells in bioresorbable nerve guides to promote and accelerate axonal regeneration. Brain Res. 2003;963(1-2):321-326.

6. Fansa H, Keilhoff G, Förster G, Seidel B, Wolf G, Schneider W. Acellular muscle with Schwann-cell implantation: an alternative biologic nerve conduit. J Reconstr Microsurg. 1999;15(7):531-537.

7. He J, Ding WL, Li F, Xia R, Wang WJ, Zhu H. Panaxydol treatment enhances the biological properties of Schwann cells in vitro. Chem Biol Interact. 2009;177(1):34-39.

8. Kim S, Im WS, Kang L, Lee ST, Chu K, Kim BI. The application of magnets directs the orientation of neurite outgrowth in cultured human neuronal cells. J Neurosci Methods. 2008;174(1):91-96.

9. Bai WF, Xu WC, Feng Y, et al. Fifty-Hertz electromagnetic fields facilitate the induction of rat bone mesenchymal stromal cells to differentiate into functional neurons. Cytotherapy. 2013;15(8):961-970.

10. Ma J, Zhang Z, Su Y, et al. Magnetic stimulation modulates structural synaptic plasticity and regulates BDNF-TrkB signal pathway in cultured hippocampal neurons. Neurochem Int. 2013;62(1):84-91.

11. Sapir Y, Cohen S, Friedman G, Polyak B. The promotion of in vitro vessel-like organization of endothelial cells in magnetically responsive alginate scaffolds. Biomaterials. 2012;33(16):4100-4109.

12. Sapir Y, Polyak B, Cohen S. Cardiac tissue engineering in magnetically actuated scaffolds. Nanotechnology. 2014;25(1):014009.
13. Meng J, Xiao B, Zhang Y, et al. Super-paramagnetic responsive nanofibrous scaffolds under static magnetic field enhance osteogenesis for bone repair in vivo. Sci Rep. 2013;3:2655.

14. Eguchi Y, Ogiue-Ikeda M, Ueno S. Control of orientation of rat Schwann cells using an 8-T static magnetic field. Neurosci Lett. 2003;351(2):130-132.

15. Riggio C, Calatayud MP, Hoskins C, et al. Poly-l-lysine-coated magnetic nanoparticles as intracellular actuators for neural guidance. Int J Nanomedicine. 2012;7:3155-3166.

16. Oh SH, Park IK, Kim JM, Lee JH. In vitro and in vivo characteristics of PCL scaffolds with pore size gradient fabricated by a centrifugation method. Biomaterials. 2007;28(9):1664-1671.

17. Mey J, Schrage K, Wessels I, Vollpracht-Crijns I. Effects of inflammatory cytokines IL-1 $\beta$, IL- 6 , and TNF $\alpha$ on the intracellular localization of retinoid receptors in Schwann cells. Glia. 2007;55(2):152-164.

18. Zhu S, Ge J, Wang Y, et al. A synthetic oxygen carrier-olfactory ensheathing cell composition system for the promotion of sciatic nerve regeneration. Biomaterials. 2014;35(5):1450-1461.

19. Zhang Y, Ding J, Duan W. A study of the effects of flux density and frequency of pulsed electromagnetic field on neurite outgrowth in PC12 cells. J Biol Phys. 2006;32(1):1-9.

20. Trillo MA, Martínez MA, Cid MA, Ubeda A. Retinoic acid inhibits the cytoproliferative response to weak $50-\mathrm{Hz}$ magnetic fields in neuroblastoma cells. Oncol Rep. 2013;29(3):885-894.

21. Cornell RM, Schwertmann U. The Iron Oxides: Structure, Properties, Reactions, Occurrences and Uses. Hoboken (NJ): Wiley; 2003.

22. Bautista MC, Bomati-Miguel O, Zhao X, et al. Comparative study of ferrofluids based on dextran-coated iron oxide and metal nanoparticles for contrast agents in magnetic resonance imaging. Nanotechnology. 2004;15(4):S154-S159.

23. Lu J, Yang S, Ng KM, et al. Solid-state synthesis of monocrystalline iron oxide nanoparticle based ferrofluid suitable for magnetic resonance imaging contrast application. Nanotechnology. 2006;17(23): 5812-5820.

24. Gupta AK, Gupta M. Synthesis and surface engineering of iron oxide nanoparticles for biomedical applications. Biomaterials. 2005;26(18):3995-4021.

25. Pilakka-Kanthikeel S, Atluri VS, Sagar V, Saxena SK, Nair M. Targeted brain derived neurotropic factors (BDNF) delivery across the blood-brain barrier for neuro-protection using magnetic nanocarriers: an in-vitro study. PLoS One. 2013;8(4):e62241.

26. Crompton KE, Goud JD, Bellamkonda RV, et al. Polylysine-functionalised thermoresponsive chitosan hydrogel for neural tissue engineering. Biomaterials. 2007; 28(3):441-449.

27. Mao JS, Zhao LG, Yin YJ, Yao KD. Structure and properties of bilayer chitosan-gelatin scaffolds. Biomaterials. 2003;24(6):1067-1074.

28. Tampieri A, Landi E, Valentini F, et al. A conceptually new type of bio-hybrid scaffold for bone regeneration. Nanotechnology. 2011;22(1):015104.

29. Goldner JS, Bruder JM, Li G, Gazzola D, Hoffman-Kim D. Neurite bridging across micropatterned grooves. Biomaterials. 2006;27(3): 460-472.

30. Kim YT, Haftel VK, Kumar S, Bellamkonda RV. The role of aligned polymer fiber-based constructs in the bridging of long peripheral nerve gaps. Biomaterials. 2008;29(21):3117-3127.

31. Bozkurt A, Brook GA, Moellers S, et al. In vitro assessment of axonal growth using dorsal root ganglia explants in a novel three-dimensional collagen matrix. Tissue Eng. 2007;13(12):2971-2979.

32. Bozkurt A, Deumens R, Beckmann C, et al. In vitro cell alignment obtained with a Schwann cell enriched microstructured nerve guide with longitudinal guidance channels. Biomaterials. 2009;30(2): 169-179.

33. Derby B. Printing and prototyping of tissues and scaffolds. Science. 2012;338(6109):921-926.

34. Terenghi G. Peripheral nerve injury and regeneration. Histol Histopathol. 1995;10(3):709-718.

35. Mirsky R, Jessen KR, Brennan A, et al. Schwann cells as regulators of nerve development. J Physiol Paris. 2002;96(1-2):17-24. 
36. Guénard V, Kleitman N, Morrissey TK, Bunge RP, Aebischer P. Syngeneic Schwann cells derived from adult nerves seeded in semipermeable guidance channels enhance peripheral nerve regeneration. J Neurosci. 1992;12(9):3310-3320.

37. Mosahebi A, Woodward B, Wiberg M, Martin R, Terenghi G. Retroviral labeling of Schwann cells: in vitro characterization and in vivo transplantation to improve peripheral nerve regeneration. Glia. 2001;34(1):8-17.

38. Rodriguez AM, Pisani D, Dechesne CA, et al. Transplantation of a multipotent cell population from human adipose tissue induces dystrophin expression in the immunocompetent mdx mouse. J Exp Med. 2005;201(9):1397-1405.

39. Zhang HY, Jin XB, Lue TF. Three important components in the regeneration of the cavernous nerve: brain-derived neurotrophic factor, vascular endothelial growth factor and the JAK/STAT signaling pathway. Asian J Androl. 2011;13(2):231-235.

40. Henderson CE, Phillips HS, Pollock RA, et al. GDNF: a potent survival factor for motoneurons present in peripheral nerve and muscle. Science. 1994;266(5187):1062-1064.
41. Woolley AG, Tait KJ, Hurren BJ, Fisher L, Sheard PW, Duxson MJ. Developmental loss of NT-3 in vivo results in reduced levels of myelin-specific proteins, a reduced extent of myelination and increased apoptosis of Schwann cells. Glia. 2008;56(3):306-317.

42. Haninec P, Kaiser R, Bobek V, Dubový P. Enhancement of musculocutaneous nerve reinnervation after vascular endothelial growth factor (VEGF) gene therapy. BMC Neurosci. 2012;13:57.

43. Sondell M, Lundborg G, Kanje M. Vascular endothelial growth factor stimulates Schwann cell invasion and neovascularization of acellular nerve grafts. Brain Res. 1999;846(2):219-228.

44. Hoyng SA, De Winter F, Gnavi S, et al. A comparative morphological, electrophysiological and functional analysis of axon regeneration through peripheral nerve autografts genetically modified to overexpress BDNF, CNTF, GDNF, NGF, NT3 or VEGF. Exp Neurol. 2014;261:578-593.
International Journal of Nanomedicine

\section{Publish your work in this journal}

The International Journal of Nanomedicine is an international, peerreviewed journal focusing on the application of nanotechnology in diagnostics, therapeutics, and drug delivery systems throughou the biomedical field. This journal is indexed on PubMed Central,

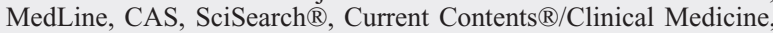

\section{Dovepress}

Journal Citation Reports/Science Edition, EMBase, Scopus and the Elsevier Bibliographic databases. The manuscript management system is completely online and includes a very quick and fair peer-review system, which is all easy to use. Visit http://www.dovepress.com/ testimonials.php to read real quotes from published authors. 